\title{
Aspectos dinâmicos de um potencial gravitacional com termo de quadrupolo
}

\author{
Rafael Cizeski Nitchai
}

\author{
DisSERTAÇÃO APRESENTADA \\ $\mathrm{AO}$ \\ Instituto De Matemática e EstatísticA \\ DA \\ Universidade DE SÃo PaUlo \\ PARA \\ OBTENÇÃO DO TÍTULO \\ DE \\ Mestre em CiÊnCIAS \\ Programa: Matemática Aplicada \\ Orientador: Prof. Dr. Frank Michael Forger
}

Durante o desenvolvimento deste trabalho o autor recebeu auxílio financeiro do CNPq

São Paulo, janeiro de 2020 


\section{Aspectos dinâmicos de um potencial gravitacional com termo de quadrupolo}

Esta é a versão original da dissertação elaborada pelo candidato Rafael Cizeski Nitchai, tal como submetida à Comissão Julgadora. 
À memória de João Cizeski. 


\section{Agradecimentos}

Em primeiro lugar, agradeço ao meu orientador, Prof. Dr. Frank Michael Forger, por todo o apoio ao longo dos últimos anos, sem o qual, certamente, a realização deste trabalho não teria sido possível.

Agradeço também ao Prof. Dr. Clodoaldo Grotta Ragazzo pelas diversas discussões e contribuições que levaram ao desenvolvimento da última seção desta dissertação.

Agradeço ao Prof. Dr. Alberto Saa, Prof. Dr. Pedro Peixoto e Prof. Dr. Salvador Addas Zanata pelas sugestões.

Agradeço ao CNPq pelo auxílio financeiro.

Finalmente, agradeço à Olivia Saa, a quem devo tudo. 


\section{Resumo}

NITCHAI, R. C. Aspectos dinâmicos de um potencial gravitacional com termo de quadrupolo, 41 f. Dissertação (Mestrado) - Instituto de Matemática e Estatística, Universidade de São Paulo, São Paulo, 2020.

Este trabalho é dividido em duas partes. No primeiro capítulo, calculamos o potencial gravitacional com termo de quadrupolo gerado por um esferoide oblato homogêneo e, em seguida, estudamos a dinâmica de uma partícula pontual orbitando tal objeto. Usando coordenadas cilíndricas, reduzimos o problema a um sistema hamiltoniano com dois graus de liberdade, cujas soluções analisamos por integração numérica para identificar aspectos qualitativos pertinentes. No segundo capítulo, aproveitamos o fato de que o referido sistema hamiltoniano possui um equilíbrio estável e outro do tipo centro-sela, assim como, no plano equatorial, uma órbita homoclínica ao último, e investigamos o comportamento das soluções próximas a esta.

Palavras-chave: esferoide oblato, potencial com momento quadrupolo, órbita homoclínica. 


\section{Abstract}

NITCHAI, R. C. Dynamical aspects of a gravitational potential with a quadrupole term, 41 pp. Dissertation (Master) - Instituto de Matemática e Estatística, Universidade de São Paulo, São Paulo, 2020.

This work is divided into two parts. In the first chapter, we calculate the gravitational potential with a quadrupole term generated by a homogeneous oblate spheroid and, in the sequel, study the dynamics of a point particle orbiting such an object. Using cyllindrical coordinates, we reduce the problem to a hamiltonian system with two degrees of freedom, whose solutions we analyze by numerical integration in order to identify pertinent qualitative aspects. In the second chapter, we take advantage of the fact that the same hamiltonian system has a stable equilibrium plus an equilibrium of saddle-center type, as well as an orbit homoclinic to the latter, and we investigate the behaviour of the solutions close to it.

Keywords: oblate spheroid, potential with quadrupole term, homoclinic orbit. 


\section{Sumário}

$\begin{array}{ll}\text { Lista de Figuras } & \text { ix }\end{array}$

$\begin{array}{ll}\text { Introdução } & 1\end{array}$

1 Potencial Gravitacional $\quad 3$

1.1 A expansão do potencial gravitacional . . . . . . . . . . . . . . . . . . . 4

1.1.1 Esfera homogênea . . . . . . . . . . . . . . . . . . 5

1.1.2 Esferoide oblato homogêneo . . . . . . . . . . . . . . . . . . . 7

1.2 Potencial Efetivo . . . . . . . . . . . . . . . . . . . . . 9

1.3 O momento angular e a inclinação orbital . . . . . . . . . . . . . . . . . . 15

1.4 Estudo numérico das órbitas . . . . . . . . . . . . . . . . . . . 16

1.5 Sobre o fenômeno de achatamento . . . . . . . . . . . . . . . . . . 19

2 Órbitas especiais $\quad 21$

2.1 Órbita cirular e a aproximação epicíclica . . . . . . . . . . . . . . . . . . . . . . . . . . . . . . . . . . . 22

2.1 .1 Variações nos momentos . . . . . . . . . . . . . . . . . . . . . . 23

2.1 .2 Variações em $r \ldots \ldots \ldots \ldots \ldots \ldots \ldots$

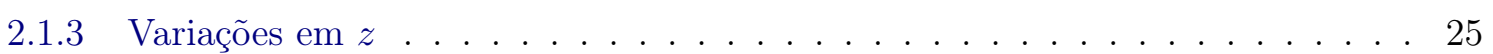

2.2 Órbita homoclínica . . . . . . . . . . . . . . . . . 26

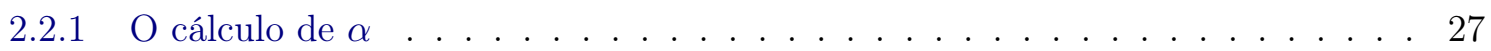

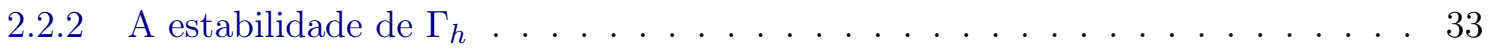

$2.2 .3 \quad$ A estabilidade de $\mathbf{0}$ por $F_{E} \neq F_{h} \ldots \ldots \ldots \ldots$

2.2 .4 Resultados numéricos . . . . . . . . . . . . . . . . . 35

2.2 .5 Escape . . . . . . . . . . . . . . . . . . . . . . . 37

3 Conclusões $\quad 39$

$\begin{array}{ll}\text { Referências Bibliográficas } & 41\end{array}$ 


\section{Lista de Figuras}

1.1 Os gráficos de $\Phi_{\text {eff }}(r, 0)$ para valores de $l$ dados, respectivamente, por: $l^{4}=e^{2}, l^{4}=$ $7.9 e^{2} / 5$ e $l^{4}=8.3 e^{2} / 5$. O parâmetro $e=0.431658$ utilizado é correspondente a elipsidade de Saturno. . . . . . . . . . . . . . . . . . . . . . . . . 13

1.2 Figura que ilustra o experimento da bala de canhão de Newton, encontrada na página 6 do livro Philosophiæ Naturalis Principia Mathematica Volume 3, De mundi systemate. 14

1.3 Soluções numéricas no espaço de configurações $(r, z)$, em função da condição inicial $\mathrm{CI}=\left(r_{0}, z_{0}, p_{r, 0}, p_{z, 0}\right)$. Figuras na mesma linha correspondem a variações na condição inicial $r_{0}$, figuras na mesma coluna correspondem a variações na condição inicial $z_{0}$. A curva vermelha corresponde à solução obtida com as mesmas condições iniciais no caso esférico $(e=0)$, e a curva preta delineia o bordo da bacia permitida do potencial efetivo, caracterizada pela equação $\Phi_{\text {eff }}(r, z)=E$. O ponto azul representa a condição inicial e o vermelho representa a posição $\left(r_{+}, 0\right)$ do mínimo do potencial efetivo. Os valores dos demais parâmetros utilizados foram $E=-0.15, e=0.10 \mathrm{e}$ $l_{z}=1.05$.

1.4 Soluções numéricas no espaço de configurações $(r, z)$, em função da condição inicial $\mathrm{CI}=\left(r_{0}, z_{0}, p_{r, 0}, p_{z, 0}\right)$. Figuras na mesma linha correspondem a variações na condição inicial $r_{0}$, figuras na mesma coluna correspondem a variações na condição inicial $z_{0}$. A curva vermelha corresponde à solução obtida com as mesmas condições iniciais no caso esférico $(e=0)$, e a curva preta delineia o bordo da bacia permitida do potencial efetivo, caracterizada pela equação $\Phi_{\text {eff }}(r, z)=E$. O ponto azul representa a condição inicial e o vermelho representa a posição $\left(r_{+}, 0\right)$ do mínimo do potencial efetivo. Os valores dos demais parâmetros utilizados foram $E=-0.15, e=0.43$ (o valor de Saturno) e $l_{z}=1.05$. . . . . . . . . . . . . . . . . . . . . . . . 18

1.5 Diagrama representando o valor do ângulo $\alpha$ como função do tempo. A Linha azul corresponde ao caso perturbado, enquanto a vermelha ao caso esférico. Os parâmetros utilizados foram $E=-0.15, e=0.43, l_{z}=1.05$ com condições iniciais $(1.5,1.5,0.27,-0.27)$ e tempo final $t=1000$. . . . . . . . . . . . . . . . . 19

1.6 Diagrama representando o valor do ângulo $\alpha$ como função do tempo. A Linha azul corresponde ao caso perturbado, enquanto a vermelha ao caso esférico. Os parâmetros utilizados foram $E=-0.15, e=0.43, l_{z}=1.05$ com condições iniciais $(1.5,1.5,0.27,-0.27)$ e tempo final $t=10000$ 
2.1 O retrato de fases de soluções no plano equatorial $\left(z=p_{z}=0\right.$ para todo $\left.t \in \mathbb{R}\right)$. $\mathrm{O}$ ponto vermelho representa o equilíbrio $\mathbf{r}_{-}$, o ponto verde representa o equilíbrio $\mathbf{r}_{+}$. As curvas destacadas em vermelho são as órbitas com energia dadas por $\Phi_{\text {eff }}\left(r_{-}, 0\right)$ e em verde por $\Phi_{\text {eff }}\left(r_{+}, 0\right)$. As legendas de cada uma das subfiguras indicam os valores de $l^{2}$. A elipsidade utilizada foi a de Saturno, $e=0.43$.

2.2 A projeção no plano $(r, z)$ da solução aproximada (em vermelho) e da solução numérica (em azul) para condições iniciais $u_{0}=\left(r_{+}, p_{r, 0}, 0, p_{z, 0}\right)$. A curva preta representa a curva equipotencial. As legendas de cada uma das subfiguras indicam os valores de $p_{r, 0}$ e $p_{z, 0}$. As constantes utilizadas foram $e=0$ e $l^{2}=2\left(1+3 e^{2} / 10\right)$

2.3 A projeção no plano $(r, z)$ da solução aproximada (em vermelho) e da solução numérica (em azul) para condições iniciais $u_{0}=\left(r_{+}+r_{0}, 10^{-2}, 0,10^{-2}\right)$. A curva preta representa a curva equipotencial. As legendas de cada uma das subfiguras indicam os valores de $r_{0}$. As constantes utilizadas foram $e=0$ e $l^{2}=2\left(1+3 e^{2} / 10\right) \ldots \ldots$.

2.4 A projeção no plano $(r, z)$ da solução aproximada (em vermelho) e da solução numérica (em azul) para condições iniciais $u_{0}=\left(r_{+}, 10^{-2}, z_{0}, 10^{-2}\right)$. A curva preta representa a curva equipotencial. As legendas de cada uma das subfiguras indicam os valores de $z_{0}$. As constantes utilizadas foram $e=0$ e $l^{2}=2\left(1+3 e^{2} / 10\right) \ldots \ldots . \quad 25$

2.5 Os gráficos de $f(\gamma)=\gamma|B|$ e $g(\gamma)=2^{-3 / 2} \ldots \ldots \ldots \ldots \ldots$

2.6 Iterações pelos mapas $F_{E}$ (esquerda) e $f_{E}$ (direita) de condições iniciais tomadas sob o eixo $\theta=\pi / 2$ da seção de Poincaré $\Sigma_{E}$. Parâmetros utilizados: $E \approx-0.4227$,

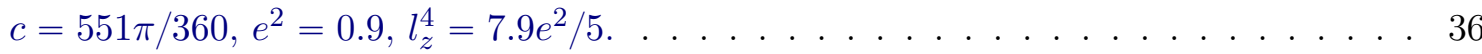

2.7 Iterações pelo mapa $F_{E}$ de condições iniciais tomadas sob o eixo $\theta=\pi / 2$ da seção de Poincaré $\Sigma_{E}$. Parâmetros utilizados: $E=h-e^{((c-5 \pi) / \gamma)}, c=551 \pi / 360, e^{2}=0.9$, $l_{z}^{4}=7.9 e^{2} / 5$.

2.8 Iterações pelos mapa $f_{E}$ de condições iniciais tomadas sob o eixo $\theta=\pi / 2$ da seção de Poincaré $\Sigma_{E}$. Parâmetros utilizados: $E=-0.40273559884568557,1=10, e^{2} / 10=$

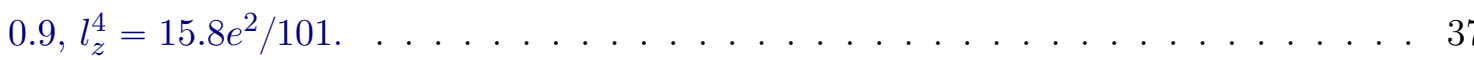




\section{Introdução}

É comum observarmos no universo estruturas celestes em forma de discos. Muitas vezes, tais objetos são constituídos por matéria difusa orbitando grandes massas centrais, como estrelas, estrelas de nêutron ou buracos negros. Este é o caso, em particular, dos discos de acreção. A origem e formação de tais objetos vêm sido investigadas ao longo dos últimos anos por diversos físicos e matemáticos. Podemos citar, por exemplo, os trabalhos de Pringle [Pri03], Milder [Mil66], e Bell e Pringle [LBP74].

Além da enorme força gravitacional exercida pelo corpo central sobre a matéria ao seu redor, as principais teorias levam em conta a conservação do momento angular total e efeitos dissipativos associados às interações entre as partículas que constituem o disco.

Porém, não é incomum a existência de figuras semelhantes a discos em condições muito menos extremas. Os anéis de Saturno são um exemplo disso. Mais ainda, a forma achatada do nosso próprio sistema solar, onde há pouca - ou nenhuma - interação entre os corpos, sugere que tal fenômeno de achatamento possa ser observado em sistemas mecânicos realistas mais simples.

Seria possível observar tal fenômeno em um sistema composto apenas por um ponto material orbitando uma massa central? Tal questionamento foi o ponto de partida deste trabalho.

No caso mais simples (isto é, para o potencial de Kepler, que é central e consequentemente integrável) todas as órbitas estão confinadas ao plano ortogonal ao momento angular da partícula - que é uma quantidade conservada. Dessa forma, este potencial pode sustentar uma estrutura de disco caso as condições iniciais sejam favoráveis, mas não é capaz de induzir a formação espontânea de uma estrutura deste tipo. Por outro lado, a introdução do momento quadrupolo pode quebrar a integrabilidade do sistema, como mostram Depetri e Saa [DS15]. Este tipo de perturbação é frequente em corpos celestes em rotação que tendem a ter raio equatorial maior que o polar e a influência de tais perturbações em situações astrofísicas envolvendo discos tem sido estudada na literatura. É neste contexto que este trabalho se insere.

No primeiro capítulo começamos nossa investigação utilizando a expansão em multipolos do potencial gravitacional. Calculamos o potencial associado aos termos de monopolo, dipolo e quadrupolo para uma distribuição de massa que representa esferoides oblatos. A seguir, utilizando um sistema de coordenadas adequadamente normalizadas, reduzimos o problema a um sistema hamiltoniano com dois graus de liberdade no qual as equações de movimento são dadas pelo gradiente de um novo potencial, chamado de potencial efetivo. Este novo sistema nos fornece algumas informações a respeito da dinâmica do problema; porém, sem uma solução explícita não é possível obter uma resposta concreta a respeito de um possível fenômeno de achatamento. Portanto, empregamos métodos computacionais para calcular soluções numéricas das equações de movimento, buscando assim identificar traços qualitativos do seu comportamento, inclusive no que diz respeito ao papel da perturbação na dinâmica e se, de alguma forma, esta poderia, por si só, dar origem a uma estrutura de disco.

No segundo capítulo nos concentramos em estudar certas órbitas especiais no retrato de fase, associadas aos pontos de máximo e de mínimo local do potencial efetivo, restrito ao plano equatorial. O ponto de mínimo, que permanece mínimo mesmo após reinserção da variável transversal ao plano equatorial e portanto é um equilíbrio estável do sistema hamiltoniano, representa uma órbita circular, e em sua vizinhança é possível obter soluções aproximadas para as equações de movimento, conforme o método tradicional de pequenas oscilações. Por outro lado, o ponto de máximo, que se 
torna um ponto crítico do tipo centro-sela após reinserção da variável transversal ao plano equatorial, possui características semelhantes às de outros sistemas hamiltonianos com dois graus de liberdade já estudados na literatura, como por exemplo em Zanata e Ragazzo [ZR01] e Figueiredo et al. [dFRM98]. A semelhança entre estes sistemas é a existência de uma órbita especial no retrato de fase que é homoclínica ao equilíbrio centro-sela. Resultados obtidos em Ragazzo [Rag97b] nos fornecem informações a respeito da dinâmica local do sistema próximo a tal solução. Porém, vale destacar que para o caso do potencial gravitacional em questão ser gerado por um esferoide oblato, o equilíbrio centro-sela e pelo menos parte da órbita homoclínica estão sempre situados em regiões do espaço de configurações ocupadas pelo corpo central, obviamente desafiando a relevância física de resultados matemáticos a respeito.

Os códigos utilizados para obter os resultados numéricos podem ser encontrados no link: https: //github.com/rnitchai/mestrado. 


\section{Capítulo 1}

\section{Potencial Gravitacional}

Considere um sistema composto por dois pontos materiais de massa $m_{1}$ e $m_{2}$, localizados, respectivamente, em pontos $\mathbf{u}_{1}$ e $\mathbf{u}_{2}$ de $\mathbb{R}^{3}$. A força gravitacional exercida pelo corpo de massa $m_{1}$ sobre o corpo de massa $m_{2}$ é dada por

$$
\mathbf{F}_{12}=-\frac{G m_{1} m_{2}}{\left|\mathbf{u}_{\mathbf{2}}-\mathbf{u}_{\mathbf{1}}\right|^{3}}\left(\mathbf{u}_{\mathbf{2}}-\mathbf{u}_{\mathbf{1}}\right)
$$

e definindo

$$
U_{\mathrm{s}}(\mathbf{v})=-\frac{G m_{1}}{\left|\mathbf{v}-\mathbf{u}_{\mathbf{1}}\right|}
$$

segue da segunda Lei de Newton que

$$
\ddot{\mathbf{r}}_{2}=-\frac{G m_{1}}{\left|\mathbf{u}_{2}-\mathbf{u}_{1}\right|^{3}}\left(\mathbf{u}_{2}-\mathbf{u}_{1}\right)=-\nabla U_{\mathrm{s}}\left(\mathbf{u}_{2}\right) .
$$

Nestas condições, dizemos que $U_{\mathrm{s}}$ é o potencial gravitacional Newtoniano associado ao corpo pontual de massa $m_{1}$.

Agora considere um conjunto de corpos pontuais isolados de massas $m_{1}, m_{2}, \ldots, m_{n}$ localizadas em $u_{1}, u_{2}, \ldots, u_{n} \in \mathbb{R}^{3}$, respectivamente. A força gravitacional exercida sobre a partícula de massa $m_{i}$ pelo restante do sistema é dada pela justaposição das forças individuais exercidas sobre $m_{i}$ por cada uma das partículas de massa $m_{j}$, onde $j=1,2, \ldots, i-1, i+1, \ldots, n$. Explicitamente, se $F_{i}$ é a força gravitacional agindo sobre a partícula de massa $m_{i}$, então

$$
F_{i}=\sum_{\substack{j=1 \\ j \neq i}}^{n} F_{j i}=-\sum_{\substack{j=1 \\ j \neq i}}^{n} \frac{G m_{j} m_{i}}{\left|\mathbf{u}_{\mathbf{i}}-\mathbf{u}_{\mathbf{j}}\right|^{3}}\left(\mathbf{u}_{\mathbf{i}}-\mathbf{u}_{\mathbf{j}}\right) .
$$

De forma análoga ao que fizemos no caso anterior, definindo

$$
U_{\mathrm{m}}(\mathbf{v})=-\sum_{\substack{j=1 \\ j \neq i}}^{n} \frac{G m_{j}}{\left|\mathbf{v}-\mathbf{u}_{\mathbf{j}}\right|},
$$

segue que

$$
\ddot{\mathbf{r}}_{\mathbf{i}}=-\sum_{\substack{j=1 \\ j \neq i}}^{n} \frac{G m_{j}}{\left|\mathbf{u}_{\mathbf{i}}-\mathbf{u}_{\mathbf{j}}\right|^{3}}\left(\mathbf{u}_{\mathbf{i}}-\mathbf{u}_{\mathbf{j}}\right)=-\nabla U_{\mathrm{m}}\left(\mathbf{u}_{\mathbf{i}}\right)
$$

e dizemos que $U_{\mathrm{m}}$ é o potencial gravitacional Newtoniano associado ao conjunto de corpos pontuais de massa $m_{j}$, com $j=1,2, \ldots, i-1, i+1, \ldots, n$.

Com isso a generalização para o caso de um sistema isolado composto por um ponto material de massa $m$ localizado em $\mathbf{x} \in \mathbb{R}^{3}$ e um corpo representado por uma distribuição de massa contínua 
com densidade $\rho$, pode ser feita utilizando uma argumentação similar aos casos discretos. A força gravitacional agindo sobre o ponto material é dada por

$$
\begin{aligned}
\mathbf{F}(\mathbf{x}) & =-\int_{\mathbb{R}^{3}} d \mu\left(\mathbf{x}^{\prime}\right) \frac{G m}{\left|\mathbf{x}-\mathbf{x}^{\prime}\right|^{3}}\left(\mathbf{x}-\mathbf{x}^{\prime}\right) \\
& =-G m \int_{\mathbb{R}^{3}} d^{3} x \frac{\rho\left(\mathbf{x}^{\prime}\right)}{\left|\mathbf{x}-\mathbf{x}^{\prime}\right|^{3}}\left(\mathbf{x}-\mathbf{x}^{\prime}\right)
\end{aligned}
$$

e definindo

$$
\Phi(\mathbf{x})=-G \int_{\mathbb{R}^{3}} d^{3} x^{\prime} \frac{\rho\left(\mathbf{x}^{\prime}\right)}{\left|\mathbf{x}-\mathbf{x}^{\prime}\right|}
$$

segue que

$$
\ddot{\mathbf{x}}=-\nabla \Phi(x) .
$$

Diremos que $\Phi$ é o potencial gravitacional associado a distribuição de massa $\rho$.

Note que, dependendo da distribuição $\rho$, obter uma fórmula explicita para o potencial pode ser uma tarefa complexa. Neste caso, uma técnica muito utilizada é expandir o integrando de (1.8) em potencias de $|\mathbf{x}|^{-1}$. Tal série, conhecida como "expansão em multipolos", tem fórmula geral conhecida e dada em função de polinômios de Legendre. Cada termo da série recebe um nome associado a sua respectiva potência de $|\mathbf{x}|^{-1}$. O primeiro termo é chamado de "monopolo", o segundo de "dipolo", o terceiro de "quadrupolo", e assim por diante.

Neste trabalho estaremos interessados na aproximação do potencial gravitacional composta pelos termos de monopolo, dipolo e quadrupolo. Ao invés de utilizar a fórmula geral citada acima, optamos por derivar cada um dos termos explicitamente.

\subsection{A expansão do potencial gravitacional}

Considere uma distribuição de massa $\rho$ associada a um corpo com dimensões e densidade arbitrárias, porém, com massa total $M$ e seu respectivo potencial dado por (1.8). Note que

$$
\frac{1}{\left|\mathbf{x}-\mathbf{x}^{\prime}\right|}=\left(|\mathbf{x}|^{2}+\left|\mathbf{x}^{\prime}\right|^{2}-2 \mathbf{x} \cdot \mathbf{x}^{\prime}\right)^{-1 / 2}
$$

Seja $|\mathbf{x}|=R$, então

$$
\frac{1}{\left|\mathbf{x}-\mathbf{x}^{\prime}\right|}=\frac{1}{R}\left(1+\frac{\mathbf{x}^{\prime 2}}{R^{2}}-\frac{2 \mathrm{x} \cdot \mathbf{x}^{\prime}}{R^{2}}\right)^{-\frac{1}{2}} .
$$

Tomando a expansão de Taylor de $(1+x)^{n}$ em torno do 0 , segue que

$$
\begin{aligned}
\frac{1}{\left|\mathbf{x}-\mathbf{x}^{\prime}\right|} & =\frac{1}{R}\left(1-\frac{1}{2}\left[\frac{\left|\mathbf{x}^{\prime}\right|^{2}}{R^{2}}-\frac{2 \mathbf{x} \cdot \mathbf{x}^{\prime}}{R^{2}}\right]+\frac{3}{8}\left[\frac{\left|\mathbf{x}^{\prime}\right|^{2}}{R^{2}}-\frac{2 \mathbf{x} \cdot \mathbf{x}^{\prime}}{R^{2}}\right]^{2}+\mathcal{O}\left(R^{-3}\right)\right) \\
& =\frac{1}{R}+\frac{\mathbf{x} \cdot \mathbf{x}^{\prime}}{R^{3}}+\frac{1}{2 R^{3}}\left(\frac{3\left(\mathbf{x} \cdot \mathbf{x}^{\prime}\right)^{2}}{R^{2}}-\left|\mathbf{x}^{\prime}\right|^{2}\right)+\mathcal{O}\left(R^{-4}\right) .
\end{aligned}
$$

O terceiro termo do lado direito dessa equação pode ser reescrito como

$$
\begin{aligned}
\frac{3\left(\mathbf{x} \cdot \mathbf{x}^{\prime}\right)^{2}}{R^{2}}-\left|\mathbf{x}^{\prime}\right|^{2} & =\frac{3}{R^{2}} \mathbf{x}\left(\mathbf{x}^{\prime} \otimes \mathbf{x}^{\prime}\right) \mathbf{x}-\frac{\left|\mathbf{x}^{\prime}\right|^{2}}{R^{2}} \operatorname{xId}_{3 \times 3} \mathbf{x} \\
& =\frac{1}{R^{2}}\left(\mathbf{x}\left[3 \mathbf{x}^{\prime} \otimes \mathbf{x}^{\prime}-\left|\mathbf{x}^{\prime}\right|^{2} \operatorname{Id}_{3 \times 3}\right] \mathbf{x}\right) .
\end{aligned}
$$


Assim, segue que

$$
\begin{aligned}
\Phi(\mathbf{x}) \approx & -\frac{G}{R} \int_{\mathbb{R}^{3}} d^{3} x^{\prime} \rho\left(\mathbf{x}^{\prime}\right) \\
& -\frac{G}{R^{3}} \mathbf{x} \cdot \int_{\mathbb{R}^{3}} d^{3} x^{\prime} \mathbf{x}^{\prime} \rho\left(\mathbf{x}^{\prime}\right) \\
& -\frac{G}{2 R^{5}} \mathbf{x}\left(\int_{\mathbb{R}^{3}} d^{3} x^{\prime} \rho\left(\mathbf{x}^{\prime}\right)\left(3 \mathbf{x}^{\prime} \otimes \mathbf{x}^{\prime}-\left|\mathbf{x}^{\prime}\right|^{2} \operatorname{Id}_{3 \times 3}\right)\right) \mathbf{x} .
\end{aligned}
$$

O primeiro, segundo e terceiro termo do lado direito da equação acima são, respectivamente os termos de monopolo, dipolo e quadrupolo da aproximação. Note que a integral no termo dipolo é proporcional ao centro de massa do corpo. Sendo assim, sem perda de generalidade, assumindo que o centro de massa está na origem, ou seja, que

$$
\frac{\int_{\mathbb{R}^{3}} d^{3} x^{\prime} \mathbf{x}^{\prime} \rho\left(x^{\prime}\right)}{\int_{\mathbb{R}^{3}} d^{3} x^{\prime} \rho\left(x^{\prime}\right)}=0,
$$

o segundo termo de (1.14) desaparece, e portanto

$$
\Phi(\mathbf{x}) \approx-\frac{G M}{R}-\frac{G}{2 R^{5}} \mathbf{x} Q \mathbf{x},
$$

onde $Q$ é o tensor simétrico de traço nulo

$$
Q_{i j}=\int_{\mathbb{R}^{3}} d^{3} x^{\prime} \rho\left(\mathbf{x}^{\prime}\right)\left(3 x_{i}^{\prime} x_{j}^{\prime}-\left|\mathbf{x}^{\prime}\right|^{2} \delta_{i j}\right)
$$

A partir deste momento denotaremos por $\Phi$ a aproximação obtida acima. Nas próximas seções calcularemos explicitamente $\Phi$ para $\rho$ satisfazendo certas condições. Nestes cálculos o apóstrofe será omitido do vetor $\mathrm{x}$ e suas componentes $x_{i}$ e $x_{j}$.

\subsubsection{Esfera homogênea}

Considere uma esfera homogênea de raio $a$ e massa total $M$. A distribuição de massa $\rho$ que representa tal objeto é dada por

$$
\rho(\mathbf{x})= \begin{cases}k, & \text { se }|\mathbf{x}| \leqslant a \\ 0, & \text { c.c. }\end{cases}
$$

Mas sabemos que

$$
M=\int_{\mathbb{R}^{3}} d^{3} x \rho(\mathbf{x})=\int_{\mathrm{B}_{a}(0)} d^{3} x k=k \frac{4 \pi a^{3}}{3},
$$

onde $\mathrm{B}_{a}(0)=\left\{\mathbf{y} \in \mathbb{R}^{3}|| \mathbf{y} \mid \leqslant a\right\}$. E assim

$$
k=\frac{3 M}{4 \pi a^{3}} .
$$

Podemos calcular então $Q_{i j}$ utilizando cordenadas esféricas:

$$
\left\{\begin{array}{l}
x_{1}=R \sin \theta \cos \varphi \\
x_{2}=R \sin \theta \sin \varphi \\
x_{3}=R \cos \theta
\end{array}\right.
$$

$\operatorname{com} R \in[0, a], \theta \in[0, \pi]$ e $\varphi \in[0,2 \pi]$. 
Termos mistos $(i \neq j)$

A fórmula geral dos termos mistos é dada por

$$
\begin{aligned}
Q_{i j} & =\frac{9 M}{4 \pi a^{3}} \int_{\mathrm{B}_{a}(0)} d^{3} x x_{i} x_{j} \\
& =\frac{9 M}{4 \pi a^{3}} \int_{R=0}^{a} \int_{\varphi=0}^{2 \pi} \int_{\theta=0}^{\pi} d R d \varphi d \theta \quad x_{i}(R, \varphi, \theta) x_{j}(R, \varphi, \theta) R^{2} \sin \theta .
\end{aligned}
$$

Assim,

$$
\begin{aligned}
Q_{12} & =\frac{9 M}{4 \pi a^{3}} \int_{R=0}^{a} \int_{\varphi=0}^{2 \pi} \int_{\theta=0}^{\pi} d R d \varphi d \theta R^{4} \sin ^{3} \theta \cos \varphi \sin \varphi=0 . \\
Q_{13} & =\frac{9 M}{4 \pi a^{3}} \int_{R=0}^{a} \int_{\varphi=0}^{2 \pi} \int_{\theta=0}^{\pi} d R d \varphi d \theta R^{4} \sin ^{2} \theta \cos \theta \cos \varphi=0 . \\
Q_{23} & =\frac{9 M}{4 \pi a^{3}} \int_{R=0}^{a} \int_{\varphi=0}^{2 \pi} \int_{\theta=0}^{\pi} d R d \varphi d \theta R^{4} \sin ^{2} \theta \cos \theta \sin \varphi=0 .
\end{aligned}
$$

Termos puros $(i=j)$

A fórmula geral dos termos puros é dada por

$$
\begin{aligned}
Q_{i i}= & \frac{9 M}{4 \pi a^{3}} \int_{\mathrm{B}_{a}(0)} d^{3} x x_{i} x_{j}-\frac{3 M}{4 \pi a^{3}} \int_{\mathrm{B}_{a}(0)} d^{3} x|\mathbf{x}|^{2} \\
= & \frac{9 M}{4 \pi a^{3}} \int_{R=0}^{a} \int_{\varphi=0}^{2 \pi} \int_{\theta=0}^{\pi} d R d \varphi d \theta \quad x_{i}(R, \varphi, \theta) x_{j}(R, \varphi, \theta) R^{2} \sin \theta \\
& -\frac{3 M}{4 \pi a^{3}} \int_{R=0}^{a} \int_{\varphi=0}^{2 \pi} \int_{\theta=0}^{\pi} d R d \varphi d \theta R^{4} \sin \theta
\end{aligned}
$$

Assim,

$$
\begin{aligned}
Q_{11}= & \frac{9 M}{4 \pi a^{3}} \int_{R=0}^{a} \int_{\varphi=0}^{2 \pi} \int_{\theta=0}^{\pi} d R d \varphi d \theta R^{4} \sin ^{3} \theta \cos ^{2} \varphi \\
& -\frac{3 M}{4 \pi a^{3}} \int_{R=0}^{a} \int_{\varphi=0}^{2 \pi} \int_{\theta=0}^{\pi} d R d \varphi d \theta R^{4} \sin \theta \\
= & \frac{9 M}{4 \pi a^{3}}\left(\left[\frac{R^{5}}{5}\right]_{0}^{a}\left[\frac{1}{12}(\cos (3 \theta)-9 \cos (\theta))\right]_{0}^{\pi}\left[\frac{1}{2}(\varphi+\sin (\varphi) \cos (\varphi))\right]_{0}^{2 \pi}\right) \\
& -\frac{3 M}{4 \pi a^{3}}\left(\left[\frac{R^{5}}{5}\right]_{0}^{a}[-\cos (\theta)]_{0}^{\pi}[\varphi]_{0}^{2 \pi}\right) \\
= & \frac{9 M}{4 \pi a^{3}}\left(\frac{4 \pi a^{5}}{15}\right)-\frac{3 M}{4 \pi a^{3}}\left(\frac{4 \pi a^{5}}{5}\right)=0 .
\end{aligned}
$$




$$
\begin{aligned}
Q_{22}= & \frac{9 M}{4 \pi a^{3}} \int_{R=0}^{a} \int_{\varphi=0}^{2 \pi} \int_{\theta=0}^{\pi} d R d \varphi d \theta R^{4} \sin ^{3} \theta \sin ^{2} \varphi \\
& -\frac{3 M}{4 \pi a^{3}} \int_{R=0}^{a} \int_{\varphi=0}^{2 \pi} \int_{\theta=0}^{\pi} d R d \varphi d \theta R^{4} \sin \theta \\
= & \frac{9 M}{4 \pi a^{3}}\left(\left[\frac{R^{5}}{5}\right]_{0}^{a}\left[\frac{1}{12}(\cos (3 \theta)-9 \cos (\theta))\right]_{0}^{\pi}\left[\frac{1}{2}(\varphi-\sin (\varphi) \cos (\varphi))\right]_{0}^{2 \pi}\right) \\
& -\frac{3 M}{4 \pi a^{3}}\left(\left[\frac{R^{5}}{5}\right]_{0}^{a}[-\cos (\theta)]_{0}^{\pi}[\varphi]_{0}^{2 \pi}\right) \\
= & \frac{9 M}{4 \pi a^{3}}\left(\frac{4 \pi a^{5}}{15}\right)-\frac{3 M}{4 \pi a^{3}}\left(\frac{4 \pi a^{5}}{5}\right)=0 .
\end{aligned}
$$

Como $Q$ tem traço nulo, segue que $Q_{33}=0$ e, portanto, o potencial (válido na região externa ao corpo central, ou seja, quando $R>a$ ) é:

$$
\Phi(\mathbf{x})=-\frac{G M}{R} .
$$

\subsubsection{Esferoide oblato homogêneo}

Um esferoide é um elipsóide que possui dois eixos de mesmo comprimento. Sejam $a$ e $c$ os comprimentos de tamanhos diferentes, considere um esferoide centrado na origem, orientado de forma que possua simetria de rotação em relação ao eixo $x_{3}$. Assim, sua equação é dada por

$$
\frac{x_{1}^{2}}{a^{2}}+\frac{x_{2}^{2}}{a^{2}}+\frac{x_{3}^{2}}{c^{2}}=1 .
$$

Diremos que, se $a>c$, então o esferoide será oblato (achatado nos pólos).

Considere então um esferoide oblato, como descrito acima, com densidade de massa constante, eixos de comprimento $a$ e $c$ e massa total $M$. De forma análoga ao que fizemos na seção anterior, encontra-se que

$$
\rho(\mathbf{x})=\left\{\begin{array}{l}
\frac{3 M}{4 \pi a^{2} c}, \quad \text { se } \frac{x_{1}^{2}}{a^{2}}+\frac{x_{2}^{2}}{a^{2}}+\frac{x_{3}^{2}}{c^{2}} \leqslant 1 \\
0, \quad \text { c.c. }
\end{array}\right.
$$

Podemos então calcular os termos $Q_{i j}$. Note primeiramente que fazendo a mudança de variáveis

$$
\left\{\begin{array}{l}
x_{1}=\hat{x}_{1} \\
x_{2}=\hat{x}_{2} \\
x_{3}=\frac{c}{a} \hat{x}_{3}
\end{array}\right.
$$

a equação (1.28) é reescrita como

$$
\hat{x}_{1}^{2}+\hat{x}_{2}^{2}+\hat{x}_{3}^{2}=a^{2}
$$

$\log 0$

$$
\int_{\mathrm{OB}_{(a, c)}(0)} d^{3} x f(\mathbf{x})=\frac{c}{a} \int_{\mathrm{B}_{a}(0)} d^{3} \hat{x} f(\hat{\mathbf{x}}),
$$

onde $\operatorname{OB}_{(a, c)}(0)=\left\{\begin{array}{l|l}\mathbf{y} \in \mathbb{R}^{3} & \frac{y_{1}^{2}}{a^{2}}+\frac{y_{2}^{2}}{a^{2}}+\frac{y_{3}^{2}}{c^{2}} \leqslant 1\end{array}\right\}$ e $f$ é uma função integrável qualquer. 
Termos mistos $(i \neq j)$

A fórmula geral dos termos mistos é dada por

$$
Q_{i j}=\frac{9 M}{4 \pi a^{2} c} \int_{\mathrm{OB}_{(a, c)}(0)} d^{3} x x_{i} x_{j}=\frac{9 M}{4 \pi a^{3}} \int_{\mathrm{B}_{a}(0)} d^{3} \hat{x} \hat{x}_{i} \hat{x}_{j}
$$

Assim,

$$
\begin{aligned}
Q_{12} & =\frac{9 M}{4 \pi a^{3}} \int_{R=0}^{a} \int_{\varphi=0}^{2 \pi} \int_{\theta=0}^{\pi} d R d \varphi d \theta R^{4} \sin ^{3} \theta \cos \varphi \sin \varphi=0 . \\
Q_{13} & =\frac{9 M c}{4 \pi a^{2}} \int_{R=0}^{a} \int_{\varphi=0}^{2 \pi} \int_{\theta=0}^{\pi} d R d \varphi d \theta R^{4} \sin ^{2} \theta \cos \theta \cos \varphi=0 . \\
Q_{23} & =\frac{9 M c}{4 \pi a^{2}} \int_{R=0}^{a} \int_{\varphi=0}^{2 \pi} \int_{\theta=0}^{\pi} d R d \varphi d \theta R^{4} \sin ^{2} \theta \cos \theta \sin \varphi=0 .
\end{aligned}
$$

Termos puros $(i=j)$

A fórmula para os termos puros $Q_{11}$ e $Q_{22}$ é dada por

$$
\begin{aligned}
Q_{i i} & =\frac{9 M}{4 \pi a^{2} c} \int_{\mathrm{OB}_{(a, c)}(0)} d^{3} x x_{i}^{2}-\frac{3 M}{4 \pi a^{2} c} \int_{\mathrm{OB}_{(a, c)}(0)} d^{3} x|\mathbf{x}|^{2} \\
& =\frac{9 M}{4 \pi a^{3}} \int_{\mathrm{B}_{a}(0)} d^{3} \hat{x} \hat{x}_{i}^{2}-\frac{3 M}{4 \pi a^{3}} \int_{\mathrm{B}_{a}(0)} d^{3} \hat{x}\left(\hat{x}_{1}^{2}+\hat{x}_{2}^{2}+\frac{c^{2}}{a^{2}} \hat{x}_{3}^{2}\right) .
\end{aligned}
$$

Utilizando coordenadas polares, encontramos que

$$
\begin{aligned}
Q_{11}= & \frac{6 M}{4 \pi a^{3}} \int_{R=0}^{a} \int_{\varphi=0}^{2 \pi} \int_{\theta=0}^{\pi} d R d \varphi d \theta R^{4} \sin ^{3} \theta \cos ^{2} \varphi \\
& -\frac{3 M}{4 \pi a^{3}} \int_{R=0}^{a} \int_{\varphi=0}^{2 \pi} \int_{\theta=0}^{\pi} d R d \varphi d \theta R^{4} \sin ^{3} \theta \sin ^{2} \varphi \\
& -\frac{3 M c^{2}}{4 \pi a^{5}} \int_{R=0}^{a} \int_{\varphi=0}^{2 \pi} \int_{\theta=0}^{\pi} d R d \varphi d \theta R^{4} \sin \theta \cos ^{2} \theta \\
= & \frac{6 M}{4 \pi a^{3}}\left(\left[\frac{R^{5}}{5}\right]_{0}^{a}\left[\frac{1}{12}(\cos (3 \theta)-9 \cos (\theta))\right]_{0}^{\pi}\left[\frac{1}{2}(\varphi+\sin (\varphi) \cos (\varphi))\right]_{0}^{2 \pi}\right) \\
& -\frac{3 M}{4 \pi a^{3}}\left(\left[\frac{R^{5}}{5}\right]_{0}^{a}\left[\frac{1}{12}(\cos (3 \theta)-9 \cos (\theta))\right]_{0}^{\pi}\left[\frac{1}{2}(\varphi-\sin (\varphi) \cos (\varphi))\right]_{0}^{2 \pi}\right) \\
& -\frac{3 M c^{2}}{4 \pi a^{5}}\left(\left[\frac{R^{5}}{5}\right]_{0}^{a}\left[-\frac{\cos 3(\theta)}{3}\right]_{0}^{\pi}[\varphi]_{0}^{2 \pi}\right) \\
= & \frac{6 M}{4 \pi a^{3}}\left(\frac{4 \pi a^{5}}{15}\right)-\frac{3 M}{4 \pi a^{3}}\left(\frac{4 \pi a^{5}}{15}\right)-\frac{3 M c^{2}}{4 \pi a^{5}}\left(\frac{4 \pi a^{5}}{15}\right)=\frac{M a^{2}}{5}\left(1-\frac{c^{2}}{a^{2}}\right) .
\end{aligned}
$$




$$
\begin{aligned}
Q_{22}= & -\frac{3 M}{4 \pi a^{3}} \int_{R=0}^{a} \int_{\varphi=0}^{2 \pi} \int_{\theta=0}^{\pi} d R d \varphi d \theta R^{4} \sin ^{3} \theta \cos ^{2} \varphi \\
& +\frac{6 M}{4 \pi a^{3}} \int_{R=0}^{a} \int_{\varphi=0}^{2 \pi} \int_{\theta=0}^{\pi} d R d \varphi d \theta R^{4} \sin ^{3} \theta \sin ^{2} \varphi \\
& -\frac{3 M c^{2}}{4 \pi a^{5}} \int_{R=0}^{a} \int_{\varphi=0}^{2 \pi} \int_{\theta=0}^{\pi} d R d \varphi d \theta R^{4} \sin \theta \cos ^{2} \theta \\
= & -\frac{3 M}{4 \pi a^{3}}\left(\left[\frac{R^{5}}{5}\right]_{0}^{a}\left[\frac{1}{12}(\cos (3 \theta)-9 \cos (\theta))\right]_{0}^{\pi}\left[\frac{1}{2}(\varphi+\sin (\varphi) \cos (\varphi))\right]_{0}^{2 \pi}\right) \\
& +\frac{6 M}{4 \pi a^{3}}\left(\left[\frac{R^{5}}{5}\right]_{0}^{a}\left[\frac{1}{12}(\cos (3 \theta)-9 \cos (\theta))\right]_{0}^{\pi}\left[\frac{1}{2}(\varphi-\sin (\varphi) \cos (\varphi))\right]_{0}^{2 \pi}\right) \\
& -\frac{3 M c^{2}}{4 \pi a^{5}}\left(\left[\frac{R^{5}}{5}\right]_{0}^{a}\left[-\frac{\cos 3(\theta)}{3}\right]_{0}^{\pi}[\varphi]_{0}^{2 \pi}\right) \\
= & -\frac{3 M}{4 \pi a^{3}}\left(\frac{4 \pi a^{5}}{15}\right)^{2 \pi}+\frac{6 M}{4 \pi a^{3}}\left(\frac{4 \pi a^{5}}{15}\right)-\frac{3 M c^{2}}{4 \pi a^{5}}\left(\frac{4 \pi a^{5}}{15}\right)=\frac{M a^{2}}{5}\left(1-\frac{c^{2}}{a^{2}}\right) .
\end{aligned}
$$

Como $Q_{11}+Q_{22}+Q_{33}=0$, segue que

$$
Q=\frac{M e^{2} a^{2}}{5}\left(\begin{array}{lll}
1 & 0 & 0 \\
0 & 1 & 0 \\
0 & 0 & -2
\end{array}\right)
$$

onde

$$
e^{2}=\left(1-\frac{c^{2}}{a^{2}}\right)
$$

A constante $e$ é chamada elipsidade e representa numericamente o achatamento do esferoide.

Assim, encontramos a fórmula do potencial, válida na região externa ao corpo central (isto é, quando $x_{1}^{2} / a^{2}+x_{2}^{2} / a^{2}+x_{3}^{2} / c^{2}>1$, ou seja, $\left.R^{2} / a^{2}+e^{2} x_{3}^{2} /\left(1-e^{2}\right) a^{2}\right)$

$$
\begin{aligned}
\Phi(\mathbf{x}) & =-\frac{G M}{R}-\frac{G M e^{2} a^{2}}{10 R^{5}}\left(x_{1}^{2}+x_{2}^{2}-2 x_{3}^{2}\right) \\
& =-\frac{G M}{R}-\frac{G M e^{2} a^{2}}{10 R^{5}}\left(R^{2}-3 x_{3}^{2}\right),
\end{aligned}
$$

\subsection{Potencial Efetivo}

Nesta seção, motivados pela natureza axissimétrica do problema e usando conceitos elementares do formalismo hamiltoniano, expressaremos o potencial e as equações de movimento do problema em coordenadas cilíndricas, $(r, \varphi, z)$. Devido à simetria axial, encontraremos, além da energia $E$, mais uma constante de movimento, a saber, a componente do momento angular ao longo do eixo de simetria, $L_{z}$. Isso nos permitirá reduzir as equações de movimento a um sistema com apenas dois graus de liberdade no qual aparece o gradiente de um novo potencial, chamado potencial efetivo.

Começamos reescrevendo (1.40) em coordenadas cilíndricas,

$$
\Phi(r, z)=-G M\left(\frac{1}{\left(r^{2}+z^{2}\right)^{1 / 2}}+\frac{e^{2} a^{2}\left(r^{2}-2 z^{2}\right)}{10\left(r^{2}+z^{2}\right)^{5 / 2}}\right),
$$


onde $r^{2}=x_{1}^{2}+x_{2}^{2}$ e $z=x_{3}$. Dessa forma a Hamiltoniana associada é

$$
\mathcal{H}=\frac{1}{2 m}\left(p_{r}^{2}+\frac{p_{\varphi}^{2}}{r^{2}}+p_{z}^{2}\right)+m \Phi(r, z)
$$

e as equações de Hamilton são

$$
\begin{array}{llll}
\frac{d r}{d t}=\frac{p_{r}}{m} & , \frac{d \varphi}{d t}=\frac{p_{\varphi}}{m r^{2}} & , \quad \frac{d z}{d t}=\frac{p_{z}}{m} \\
\frac{d p_{r}}{d t}=\frac{p_{\varphi}^{2}}{m r^{3}}-m \partial_{r} \Phi & , \frac{d p_{\varphi}}{d t}=0 & , \frac{d p_{z}}{d t}=-m \partial_{z} \Phi .
\end{array}
$$

Vemos que $L_{z} \stackrel{\text { def }}{=} p_{\varphi}$ é uma quantidade conservada, ou seja, $\varphi$ é uma variável cíclica. Assim, definindo

$$
\Phi_{\text {eff }}(r, z) \stackrel{\text { def }}{=} \Phi(r, z)+\frac{L_{z}^{2}}{2 m^{2} r^{2}},
$$

ou seja,

$$
\Phi_{\mathrm{eff}}(r, z)=-G M\left(\frac{1}{\left(r^{2}+z^{2}\right)^{1 / 2}}+\frac{e^{2} a^{2}\left(r^{2}-2 z^{2}\right)}{10\left(r^{2}+z^{2}\right)^{5 / 2}}\right)+\frac{L_{z}^{2}}{2 m^{2} r^{2}},
$$

para reescrever a energia total na forma

$$
E=\frac{1}{2 m}\left(p_{r}^{2}+p_{z}^{2}\right)+m \Phi_{\mathrm{eff}}(r, z)
$$

podemos resolver o problema apenas no plano meridional e, com a solução em mãos, obter $\varphi(t)$ integrando a seguinte equação:

$$
\frac{d \varphi}{d t}=\frac{L_{z}}{m r^{2}} .
$$

Por isso, nosso foco estará no seguinte sistema de equações:

$$
\begin{array}{rlrl}
\frac{d r}{d t} & =\frac{p_{r}}{m} & & \frac{d z}{d t}=\frac{p_{z}}{m} \\
\frac{d p_{r}}{d t}=-m \partial_{r} \Phi_{\mathrm{eff}} & , & \frac{d p_{z}}{d t}=-m \partial_{z} \Phi_{\mathrm{eff}} .
\end{array}
$$

Para simplificar a apresentação, é conveniente reexpressar todas as quantidades de interesse em grandezas adimensionais. Para tanto, observe que as constantes $\sqrt{\mu}, a, m \mu, m a \sqrt{\mu}$ e $\sqrt{\mu} / a$ onde

$$
\mu=\frac{G M}{a},
$$

constituem quantidades características do sistema, com as respectivas dimensões de velocidade, distância, energia, momento angular e frequência para as quais daremos uma interpretação física logo abaixo e que utilizaremos para normalizar as quantidades de interesse conforme segue:

$$
\begin{gathered}
\tilde{r}=\frac{r}{a}, \quad \tilde{z}=\frac{z}{a}, \quad \tilde{t}=\frac{\sqrt{\mu}}{a} t \\
\tilde{p_{r}}=\frac{p_{r}}{m \sqrt{\mu}}, \quad \tilde{p_{z}}=\frac{p_{z}}{m \sqrt{\mu}}, \quad \tilde{E}=\frac{E}{m \mu}, \quad l_{z}=\frac{L_{z}}{m a \sqrt{\mu}}, \quad \tilde{\Phi}_{\mathrm{eff}}=\frac{\Phi_{\mathrm{eff}}}{\mu} .
\end{gathered}
$$

Dessa forma, podemos reescrever as equações (1.44)-(1.47) da seguinte forma:

$$
\tilde{\Phi}_{\mathrm{eff}}(\tilde{r}, \tilde{z})=-\frac{1}{\left(\tilde{r}^{2}+\tilde{z}^{2}\right)^{1 / 2}}-\frac{e^{2}\left(\tilde{r}^{2}-2 \tilde{z}^{2}\right)}{10\left(\tilde{r}^{2}+\tilde{z}^{2}\right)^{5 / 2}}+\frac{l_{z}^{2}}{2 \tilde{r}^{2}}
$$




$$
\begin{gathered}
\tilde{E}=\frac{1}{2}\left({\tilde{p_{r}}}^{2}+\tilde{p}_{z}^{2}\right)+\tilde{\Phi}_{\mathrm{eff}}(\tilde{r}, \tilde{z}) . \\
\frac{d \tilde{\varphi}}{d \tilde{t}}=\frac{l_{z}}{\tilde{r}^{2}} . \\
\frac{d \tilde{r}}{d \tilde{t}}=\tilde{p}_{r} \quad, \quad \frac{d \tilde{z}}{d \tilde{t}}=\tilde{p}_{z} \\
\frac{d \tilde{p_{r}}}{d \tilde{t}}=-\partial_{\tilde{r}} \tilde{\Phi}_{\mathrm{eff}} \quad, \quad \frac{d \tilde{p_{z}}}{d \tilde{t}}=-\partial_{\tilde{z}} \tilde{\Phi}_{\mathrm{eff}} .
\end{gathered}
$$

Deste ponto em diante abandonaremos o diacrítico " " com o objetivo de manter uma notação legível. Com isso, repetimos as equações anteriores para futuras citações:

$$
\begin{gathered}
\Phi(r, z)=-\frac{1}{\left(r^{2}+z^{2}\right)^{1 / 2}}-\frac{e^{2}\left(r^{2}-2 z^{2}\right)}{10\left(r^{2}+z^{2}\right)^{5 / 2}}, \\
\Phi_{\mathrm{eff}}(r, z)=-\frac{1}{\left(r^{2}+z^{2}\right)^{1 / 2}}-\frac{e^{2}\left(r^{2}-2 z^{2}\right)}{10\left(r^{2}+z^{2}\right)^{5 / 2}}+\frac{l_{z}^{2}}{2 r^{2}}, \\
E=\frac{1}{2}\left(p_{r}^{2}+p_{z}^{2}\right)+\Phi_{\mathrm{eff}}(r, z), \\
\dot{\varphi}(t)=\frac{l_{z}}{r^{2}}, \\
\dot{r}=p_{r} \quad, \quad \dot{z}=p_{z} \\
\dot{p_{r}}=-\partial_{r} \Phi_{\mathrm{eff}} \quad, \quad \dot{p_{z}}=-\partial_{z} \Phi_{\mathrm{eff} .}
\end{gathered}
$$

Note que o conjunto $\Delta=\left\{\left(r, 0, p_{r}, 0\right) \in \mathbb{R}^{4}, r \in \mathbb{R}_{+}\right.$e $\left.p_{r} \in \mathbb{R}\right\}$ (que representa o retrato de fases restrito ao plano equatorial do sistema) é invariante pelo fluxo associado a (1.58), i.e., órbitas com posição e velocidade iniciais no plano equatorial deverão se manter nele para todo $t \in \mathbb{R}$, sujeitas ao potencial efetivo simplificado

$$
\Phi_{\mathrm{eff}}(r, 0)=-\frac{1}{r}-\frac{e^{2}}{10 r^{3}}+\frac{l_{z}^{2}}{2 r^{2}}
$$

Para obtermos uma visão qualitativa da dinâmica do sistema, começamos calculando os pontos críticos do potencial efetivo, cujas primeiras derivadas são:

$$
\begin{gathered}
\partial_{r} \Phi_{\mathrm{eff}}(r, z)=\frac{r}{\left(r^{2}+z^{2}\right)^{3 / 2}}-\frac{e^{2} r}{5\left(r^{2}+z^{2}\right)^{5 / 2}}+\frac{e^{2} r\left(r^{2}-2 z^{2}\right)}{2\left(r^{2}+z^{2}\right)^{7 / 2}}-\frac{l_{z}^{2}}{r^{3}} \\
\partial_{z} \Phi_{\mathrm{eff}}(r, z)=\frac{z}{\left(r^{2}+z^{2}\right)^{3 / 2}}+\frac{2 e^{2} z}{5\left(r^{2}+z^{2}\right)^{5 / 2}}+\frac{e^{2} z\left(r^{2}-2 z^{2}\right)}{2\left(r^{2}+z^{2}\right)^{7 / 2}} .
\end{gathered}
$$

Claramente, $\partial_{z} \Phi_{\text {eff }}(r, 0)=0$ para qualquer $r$, ou seja, essa derivada se anula no plano equatorial inteiro, e a outra vale

$$
\partial_{r} \Phi_{\mathrm{eff}}(r, 0)=\frac{1}{r^{2}}+\frac{3 e^{2}}{10 r^{4}}-\frac{l_{z}^{2}}{r^{3}}
$$

que tem raízes

$$
r_{ \pm}=\frac{1}{2}\left(l_{z}^{2} \pm \sqrt{l_{z}^{4}-\frac{6 e^{2}}{5}}\right) .
$$


Note que

$$
r_{+}+r_{-}=l_{z}^{2}, \quad r_{+} r_{-}=\frac{3 e^{2}}{10}
$$

e portanto

$$
l_{z}^{2}=r_{ \pm}+\frac{3 e^{2}}{10} \frac{1}{r_{ \pm}}, \quad \sqrt{l_{z}^{4}-\frac{6 e^{2}}{5}}= \pm\left(r_{ \pm}-\frac{3 e^{2}}{10} \frac{1}{r_{ \pm}}\right) .
$$

Para decidir qual é a natureza destes pontos de equilíbrio, calculamos as segundas derivadas do potencial efetivo:

$$
\begin{gathered}
\partial_{r} \partial_{z} \Phi_{\mathrm{eff}}(r, z)=-\frac{3 r z}{\left(r^{2}+z^{2}\right)^{5 / 2}}-\frac{e^{2} r z}{\left(r^{2}+z^{2}\right)^{7 / 2}}-\frac{7 e^{2} r z\left(r^{2}-2 z^{2}\right)}{2\left(r^{2}+z^{2}\right)^{9 / 2}}, \\
\partial_{r}^{2} \Phi_{\mathrm{eff}}(r, z)=\frac{1}{\left(r^{2}+z^{2}\right)^{3 / 2}}-\frac{15 r^{2}+e^{2}}{5\left(r^{2}+z^{2}\right)^{5 / 2}}+\frac{e^{2}\left(5 r^{2}-2 z^{2}\right)}{2\left(r^{2}+z^{2}\right)^{7 / 2}}-\frac{7 e^{2} r^{2}\left(r^{2}-2 z^{2}\right)}{2\left(r^{2}+z^{2}\right)^{9 / 2}}+\frac{3 l_{z}^{2}}{r^{4}} \\
\partial_{z}^{2} \Phi_{\mathrm{eff}}(r, z)=\frac{1}{\left(r^{2}+z^{2}\right)^{3 / 2}}-\frac{15 z^{2}-2 e^{2}}{5\left(r^{2}+z^{2}\right)^{5 / 2}}+\frac{e^{2}\left(r^{2}-10 z^{2}\right)}{2\left(r^{2}+z^{2}\right)^{7 / 2}}-\frac{7 e^{2} z^{2}\left(r^{2}-2 z^{2}\right)}{2\left(r^{2}+z^{2}\right)^{9 / 2}} .
\end{gathered}
$$

Assim, a segunda derivada mista também se anula no plano equatorial inteiro e as outras valem:

$$
\begin{gathered}
\partial_{r}^{2} \Phi_{\mathrm{eff}}(r, 0)=-\frac{2}{r^{3}}-\frac{6 e^{2}}{5 r^{5}}+\frac{3 l_{z}^{2}}{r^{4}} \\
\partial_{z}^{2} \Phi_{\mathrm{eff}}(r, 0)=\frac{1}{r^{3}}+\frac{9 e^{2}}{10 r^{5}} .
\end{gathered}
$$

Note que a segunda derivada transversal, $\partial_{z}^{2} \Phi_{\text {eff }}(r, 0)$, é sempre positiva e decresce monotonamente com $r$. Quanto a segunda derivada radial avaliada nos pontos de equilíbrio, temos:

$$
\begin{aligned}
\partial_{r}^{2} \Phi_{\text {eff }}\left(r_{ \pm}, 0\right) & =-\frac{2}{r_{ \pm}^{3}}-\frac{6 e^{2}}{5 r_{ \pm}^{5}}+\frac{3 l_{z}^{2}}{r_{ \pm}^{4}}=\frac{1}{2 r_{ \pm}^{5}}\left(-4 r_{ \pm}^{2}+6 l_{z}^{2} r_{ \pm}-\frac{12 e^{2}}{5}\right) \\
& =\frac{1}{2 r_{ \pm}^{5}}\left(-\left[l_{z}^{2} \pm \sqrt{l_{z}^{4}-\frac{6 e^{2}}{5}}\right]^{2}+3 l_{z}^{2}\left[l_{z \pm}^{2} \sqrt{l_{z}^{4}-\frac{6 e^{2}}{5}}\right]-\frac{12 e^{2}}{5}\right) \\
& =\frac{1}{2 r_{ \pm}^{5}}\left(l_{z}^{4} \pm l_{z}^{2} \sqrt{l_{z}^{4}-\frac{6 e^{2}}{5}}-\frac{6 e^{2}}{5}\right)=\frac{\sqrt{l_{z}^{4}-\frac{6 e^{2}}{5}}}{2 r_{ \pm}^{5}}\left( \pm l_{z}^{2}+\sqrt{l_{z}^{4}-\frac{6 e^{2}}{5}}\right) \\
& = \pm \frac{\sqrt{l_{z}^{4}-\frac{6 e^{2}}{5}}}{r_{ \pm}^{4}}
\end{aligned}
$$

com o seguinte resultado simples:

$$
\partial_{r}^{2} \Phi_{\text {eff }}\left(r_{ \pm}, 0\right)=\frac{1}{r_{ \pm}^{4}}\left(r_{ \pm}-\frac{3 e^{2}}{10} \frac{1}{r_{ \pm}}\right)
$$


Além disso, calculamos o valor do potencial efetivo nos pontos críticos:

$$
\begin{aligned}
\Phi_{\mathrm{eff}}\left(r_{ \pm}, 0\right) & =-\frac{1}{r_{ \pm}}-\frac{e^{2}}{10 r_{ \pm}^{3}}+\frac{l_{z}^{2}}{2 r_{ \pm}^{2}}=\frac{1}{r_{ \pm}^{2}}\left(-r_{ \pm}-\frac{e^{2}}{10 r_{ \pm}}+\frac{l_{z}^{2}}{2}\right) \\
& =\frac{1}{r_{ \pm}^{2}}\left(-r_{ \pm}-\frac{e^{2}}{10 r_{ \pm}}+\frac{r_{+}+r_{-}}{2}\right)=\frac{1}{r_{ \pm}^{2}}\left(-\frac{r_{ \pm}}{2}-\frac{e^{2}}{10 r_{ \pm}}+\frac{r_{\mp}}{2}\right) \\
& =\frac{1}{r_{ \pm}^{2}}\left(-\frac{r_{ \pm}}{2}-\frac{e^{2}}{10 r_{ \pm}}+\frac{3 e^{2}}{20 r_{ \pm}}\right),
\end{aligned}
$$

que tem resultado simplificado dado por

$$
\Phi_{\text {eff }}\left(r_{ \pm}, 0\right)=-\frac{1}{2 r_{ \pm}^{3}}\left(r_{ \pm}^{2}-\frac{e^{2}}{10}\right) .
$$

Note que $\Phi_{\text {eff }}\left(r_{-}, 0\right)=0$ se, e somente se, $r_{-}^{2}=e^{2} / 10$. Neste caso

$$
r_{+}^{2}=\left(\frac{3 e^{2}}{10}\right)^{2} \frac{10}{e^{2}}=\frac{9 e^{4}}{10}
$$

e assim

$$
l_{z}^{2}=\frac{3 e}{\sqrt{10}}+\frac{e}{\sqrt{10}}=\frac{4 e}{\sqrt{10}}
$$

ou equivalentemente $l_{z}^{4}=8 e^{2} / 5$. Sendo assim, podemos diferenciar três casos principais:

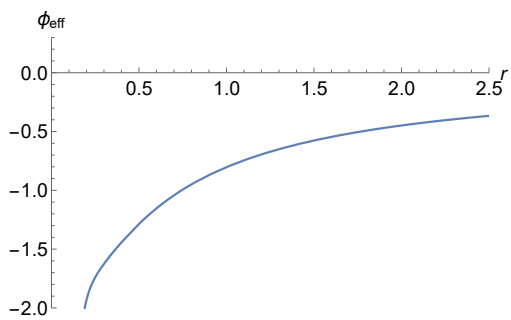

(a) $\quad l_{z}^{4}<\frac{6 e^{2}}{5}$

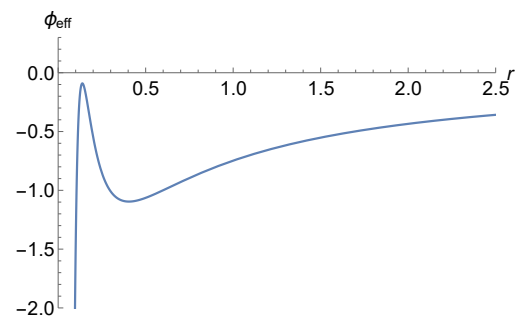

(b) $\quad \frac{6 e^{2}}{5}<l_{z}^{4}<\frac{8 e^{2}}{5}$

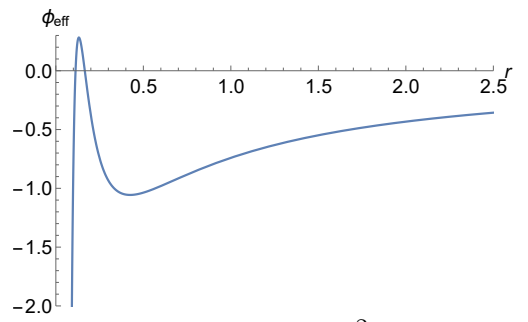

(c) $\quad l_{z}^{4}>\frac{8 e^{2}}{5}$

Figura 1.1: Os gráficos de $\Phi_{\text {eff }}(r, 0)$ para valores de $l$ dados, respectivamente, por: $l^{4}=e^{2}, l^{4}=7.9 e^{2} / 5 e$ $l^{4}=8.3 e^{2} / 5$. O parâmetro $e=0.431658$ utilizado é correspondente a elipsidade de Saturno.

Em (a) não existem pontos fixos reais e toda trajetória com energia negativa vai a zero com o passar do tempo. Já em (b) e (c), existem dois equilíbrios, um instável (representado pelo ponto de máximo local, $r_{-}$) e outro estável (representado pelo ponto de mínimo local, $r_{+}$).

Podemos nos perguntar o que acontece quando tomamos o limite esférico $(e \rightarrow 0)$. Obviamente, neste caso $r_{+} \rightarrow l_{z}^{2}$ e $r_{-} \rightarrow 0$. Por outro lado, quando $e=0$, o termo cúbico inverso desaparece do potencial e a barreira centrifugal domina o potencial efetivo para $r$ pequeno, de modo que ainda encontramos um ponto de mínimo em $l_{z}^{2}$, enquanto que o ponto de máximo local desaparece.

Antes de prosseguir, queremos comentar sobre uma questão importante, a saber, a posição dos pontos de equilíbrio em relação às dimensões do esferoide. Valores de $r_{ \pm}$menores do que 1 implicam que o ponto de equilíbrio está no interior do corpo central: obviamente, tais órbitas são irrelevantes do ponto de vista físico.

Primeiro, observamos que para $l_{z}^{4} / e^{2}>6 / 5$, o que engloba os casos representados na Fig. 1.1(b) e Fig. 1.1(c), vale

$$
r_{ \pm}=\frac{e}{2}\left(l_{z}^{2} / e \pm \sqrt{l_{z}^{4} / e^{2}-6 / 5}\right)
$$


e como sempre $l_{z}^{2} / e-\sqrt{l_{z}^{4} / e^{2}-6 / 5}<2$, pois isso equivale a $\left(\sqrt{l_{z}^{4} / e^{2}-6 / 5}+2\right)^{2}=l_{z}^{4} / e^{2}-6 / 5+$ $4 \sqrt{l_{z}^{4} / e^{2}-6 / 5}+4>l^{4} / e^{2}$, o que certamente é sempre verdade, segue que o ponto de equilíbrio $\mathbf{r}_{-}$ estará sempre no interior do esferoide. O mesmo acontece com $\mathbf{r}_{+}$se nos restringirmos aos valores de $l_{z}$ representados na Fig. $1.1(\mathrm{~b})$, onde $6 / 5 \leqslant l_{z}^{4} / e^{2} \leqslant 8 / 5$. De fato:

$$
r_{+} \leqslant \frac{e}{2}(\sqrt{8 / 5}+\sqrt{2 / 5})=\frac{e}{2 \sqrt{10}}(\sqrt{16}+\sqrt{4})=\frac{3 e}{\sqrt{10}}<1 .
$$

Por outro lado, no caso representado na Fig. 1.1(c), podemos encontrar os valores de $l_{z}^{2} / e$ para os quais vale $r_{+}>1$ :

$$
\begin{aligned}
r_{+}>1 \Longrightarrow l_{z}^{2}-\left(1+\frac{3 e^{2}}{10}\right) & =r_{+}-1+\frac{3 e^{2}}{10}\left(\frac{1}{r_{+}}-1\right) \\
& =\left(r_{+}-1\right)\left(1-\frac{3 e^{2}}{10} \frac{1}{r_{+}}\right)>0 . \\
l_{z}^{2}>1+\frac{3 e^{2}}{10} \Longrightarrow r^{2}> & \frac{1}{2}\left(1+\frac{3 e^{2}}{10}+\sqrt{\left(1+\frac{3 e^{2}}{10}\right)^{2}-\frac{6 e^{2}}{5}}\right) \\
= & \frac{1}{2}\left(1+\frac{3 e^{2}}{10}+\sqrt{\left(1-\frac{3 e^{2}}{10}\right)^{2}}\right)=1
\end{aligned}
$$

Portanto a condição $r_{+}>1$ equivale a:

$$
l_{z}^{2}>1+\frac{3 e^{2}}{10}
$$

Tal desigualdade nos dá uma estimativa quantitativa do momento angular necessário para que a partícula não se choque com a superfície do corpo central.

Agora também podemos dar uma interpretação para as constantes de normalização utilizadas acima. Para tanto imaginamos que o corpo central fosse substituído por uma distribuição de massa esfericamente simétrica homogênea com mesmo raio equatorial a e mesma massa $M$ (portanto, com densidade ligeiramente menor). Então, a velocidade $\sqrt{2 \mu}$ é a velocidade de escape de qualquer partícula da superfície deste corpo (pois a energia cinética da partícula lançada com esta velocidade inicial, $m \mu$, deve ser igual a diferença de energia potencial entre o infinito e a superfície, que vale $G M m / a)$. De modo semelhante, a velocidade $\sqrt{\mu}$ é a velocidade necessária para que a partícula entre em órbita circular raspando a superfície (pois para que a partícula possa se mover nessa órbita, a força centrifugal deve compensar a força gravitacional). Assim, as demais constantes $a$, $m \mu, m a \sqrt{\mu}$ e $\sqrt{\mu} / a$ são respectivamente, o raio, a energia, o momento angular e a frequência nesta órbita especial. Historicamente, tais velocidades foram propostas por Newton como resultado de um experimento mental -conhecido como o experimento da bala de canhão- que é descrito em seu famoso livro Principia.

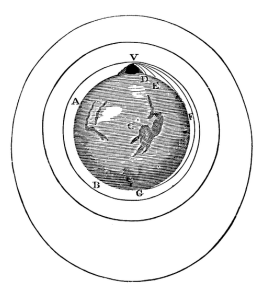

Figura 1.2: Figura que ilustra o experimento da bala de canhão de Newton, encontrada na página 6 do livro Philosophice Naturalis Principia Mathematica Volume 3, De mundi systemate. 


\subsection{O momento angular e a inclinação orbital}

Nessa seção estamos interessados em investigar o comportamento da inclinação da órbita de uma partícula sujeita ao potencial (1.54) derivado nas seções anteriores. Para isso, é necessário, antes de mais nada, definir tal quantidade. A maneira natural é identificá-la com o ângulo $\alpha$ entre o momento angular $\mathbf{l}$ da partícula e o vetor $\mathbf{e}_{z}=(0,0,1)$. Assim, o valor $\alpha=0$ corresponde ao momento angular perpendicular ao plano equatorial do sistema, o que só é possível caso os vetores posição e velocidade estejam contidos neste plano.

De modo geral, uma partícula com posição descrita em coordenadas cilíndricas normalizadas por

$$
\mathbf{r}=(r \cos \varphi, r \sin \varphi, z),
$$

e com derivada no tempo dada por

$$
\dot{\mathbf{r}}=(\dot{r} \cos \varphi-r \sin \varphi \dot{\varphi}, \dot{r} \sin \varphi+r \cos \varphi \dot{\varphi}, \dot{z})
$$

terá o momento angular normalizado dado por

$$
\mathbf{l}=\mathbf{r} \times \dot{\mathbf{r}}=\left(l_{x}, l_{y}, l_{z}\right),
$$

onde

$$
\begin{aligned}
& l_{x}=(r \sin \varphi \dot{z}-\dot{r} \sin \varphi z-r \cos \varphi \dot{\varphi} z) \\
& l_{y}=(-r \cos \varphi \dot{z}+\dot{r} \cos \varphi z-r \sin \varphi \dot{\varphi} z) \\
& l_{z}=r^{2} \dot{\varphi}
\end{aligned}
$$

Note que, definindo $\zeta=z / r$, temos

$$
l_{x}^{2}+l_{y}^{2}=(r \dot{z}-\dot{r} z)^{2}+z^{2} r^{2} \dot{\varphi}^{2}=m^{2} r^{4} \dot{\zeta}^{2}+l_{z}^{2} \zeta^{2} .
$$

Assim, podemos escrever o ângulo $\alpha$ como

$$
\cos \alpha=\frac{\mathbf{l} \cdot \mathbf{e}_{z}}{|\mathbf{l}|}=\frac{l_{z}}{|\mathbf{l}|}=\frac{1}{\sqrt{1+\left(l_{x}^{2}+l_{y}^{2}\right) / l_{z}^{2}}}=\frac{1}{\sqrt{1+\zeta^{2}+r^{4} \dot{\zeta}^{2} / l_{z}^{2}}} .
$$

Sem uma solução exata para $r$ e $z$, tal equação não nos dá nenhuma informação quantitativa a respeito do ângulo em questão. Porém, como já vimos anteriormente, $l_{z}$ é uma quantidade conservada do sistema e, portanto, qualquer variação no tempo de $\alpha$ está relacionada exclusivamente a uma variação de $|\mathbf{1}|$. Para calcularmos esta, note que

$$
\ddot{\mathbf{l}}=\mathbf{r} \times \ddot{\mathbf{r}}=-\mathbf{r} \times \nabla \Phi,
$$

mas podemos reescrever (1.54) como

$$
\Phi(\mathbf{r})=-\frac{1}{|\mathbf{r}|}-\frac{e^{2}\left(|\mathbf{r}|^{2}-3 z^{2}\right)}{10|\mathbf{r}|^{5}}
$$

e vemos que a única parte de $\Phi$ cujo gradiente não é paralelo a $\mathbf{r}$ é o termo $3 e^{2} z^{2} / 10\left(r^{2}+z^{2}\right)^{5 / 2}$ 
sendo que

$$
\begin{aligned}
& \partial_{x}\left(-3 e^{2} z^{2} / 10\left(r^{2}+z^{2}\right)^{5 / 2}\right)=-\frac{3 e^{2} x z^{2}}{2\left(r^{2}+z^{2}\right)^{7 / 2}} \\
& \partial_{y}\left(-3 e^{2} z^{2} / 10\left(r^{2}+z^{2}\right)^{5 / 2}\right)=-\frac{3 e^{2} y z^{2}}{2\left(r^{2}+z^{2}\right)^{7 / 2}} \\
& \partial_{z}\left(-3 e^{2} z^{2} / 10\left(r^{2}+z^{2}\right)^{5 / 2}\right)=\frac{6 e^{2} z}{10\left(r^{2}+z^{2}\right)^{5 / 2}}-\frac{3 e^{2} z^{3}}{2\left(r^{2}+z^{2}\right)^{7 / 2}} .
\end{aligned}
$$

Portanto, segue que

$$
\mathrm{i}=-\mathbf{r} \times \nabla \Phi=\frac{3 e^{2} z}{5\left(r^{2}+z^{2}\right)^{5 / 2}} \mathbf{e}_{z} \times \mathbf{r}
$$

e dessa forma

$$
\begin{aligned}
\frac{1}{2} \frac{d}{d t} \mathbf{l}^{2}=\mathbf{l} \cdot \mathbf{i} & =\frac{3 e^{2} z}{5\left(r^{2}+z^{2}\right)^{5 / 2}}(\mathbf{r} \times \dot{\mathbf{r}}) \cdot\left(\mathbf{e}_{\mathbf{z}} \times \mathbf{r}\right) \\
& =\frac{3 e^{2} z}{5\left(r^{2}+z^{2}\right)^{5 / 2}}\left(\left(\mathbf{r} \cdot \mathbf{e}_{\mathbf{z}}\right)(\dot{\mathbf{r}} \cdot \mathbf{r})-\left(\dot{\mathbf{r}} \cdot \mathbf{e}_{\mathbf{z}}\right)(\mathbf{r} \cdot \mathbf{r})\right) \\
& =\frac{3 e^{2} z}{5\left(r^{2}+z^{2}\right)^{5 / 2}}\left(z(r \dot{r}+z \dot{z})-\left(r^{2}+z^{2}\right) \dot{z}\right) \\
& =-\frac{3 e^{2} r z}{5\left(r^{2}+z^{2}\right)^{5 / 2}}(r \dot{z}-z \dot{r}),
\end{aligned}
$$

ou seja

$$
\frac{1}{2} \frac{d}{d t} \mathbf{l}^{2}=-\frac{3 e^{2} r^{4}}{5\left(r^{2}+z^{2}\right)^{5 / 2}} \zeta \dot{\zeta}
$$

Em particular, fica evidente que no caso de elipsidade $e=0,|\mathbf{l}|$ e $\alpha$ são constantes do movimento.

\subsection{Estudo numérico das órbitas}

A seguir pretendemos chegar a uma compreensão qualitativa das órbitas do sistema através de uma integração numérica das equações de movimento. Para tanto, estabelecemos primeiro dois critérios para a escolha das condições iniciais, que determinam $p_{r, 0}$ e $p_{z, 0}$ em termos de $r_{0}$ e $z_{0}$, em conjunto com a energia total $E$ e o momento angular $l_{z}$ como parâmetros. Note que o momento angular $l_{z}$ entra apenas na transição do potencial $\Phi$ para o potencial efetivo $\Phi_{\text {eff }}$, enquanto que conservação da energia impõe a seguinte condição sobre as condições iniciais:

$$
p_{r, 0}^{2}+p_{z, 0}^{2}=2\left(E-\Phi_{\mathrm{eff}}\left(r_{0}, z_{0}\right)\right) .
$$

Também exigimos que a posição inicial corresponda ao afélio ou periélio da órbita, que são os pontos críticos da distância $R=\sqrt{r^{2}+z^{2}}$ como função do tempo, ou seja, exigimos que

$$
\left.\frac{d}{d t} R^{2}\right|_{t=0}=2 r_{0} p_{r, 0}+2 z_{0} p_{z, 0}=0 .
$$

Os valores de $p_{r, 0}$ e $p_{z, 0}$ que satisfazem essas duas condições são

$$
p_{r, 0}= \pm \frac{\left|z_{0}\right|}{R_{0}} \sqrt{2\left(E-\Phi_{\mathrm{eff}}\left(r_{0}, z_{0}\right)\right)}, \quad p_{z, 0}=\mp \operatorname{sgn}\left(z_{0}\right) \frac{r_{0}}{R_{0}} \sqrt{2\left(E-\Phi_{\mathrm{eff}}\left(r_{0}, z_{0}\right)\right)}
$$


se $z_{0} \neq 0$, onde o sinal superior/inferior sinaliza uma velocidade radial inicial positiva/negativa, e

$$
p_{r, 0}=0, \quad p_{z, 0}= \pm \sqrt{2\left(E-\Phi_{\mathrm{eff}}\left(r_{0}, 0\right)\right)}
$$

se $z_{0}=0$, onde ambos os sinais são possíveis. Além disso, notamos que as órbitas se comportam naturalmente sob reflexão no plano equatorial: se $(r(t), z(t))$ é solução do sistema com condição inicial $\left(r_{0}, z_{0}, p_{r, 0}, p_{z, 0}\right)$, então $(r(t), z(t))$ é solução do sistema com condição inicial $\left(r_{0},-z_{0}, p_{r, 0},-p_{z, 0}\right)$. Também se comportam naturalmente sob inversão temporal: se $(r(t), z(t))$ é solução do sistema com condição inicial $\left(r_{0}, z_{0}, p_{r, 0}, p_{z, 0}\right)$, então $(r(-t),-z(-t))$ é solução do sistema com condição inicial $\left(r_{0}, z_{0},-p_{r, 0},-p_{z, 0}\right)$. Sendo assim, podemos nos restringir, nas simulações numéricas a seguir, às situações onde $z_{0} \geqslant 0, p_{r, 0} \geqslant 0$ e $p_{z, 0}<0$.

Nas Figuras 1.3 e 1.4 abaixo, exibimos as soluções, todas com energia $E=-0.15$ e com momento angular $l_{z}=1.05$, obtidas integrando o sistema até um tempo final $t=10000$ através do método de Runge-Kutta-Fehlberg de quarta ordem, para dois valores de elipsidade: $e=0.10$ (Fig. 1.3) e $e=0.43$ (Fig. 1.4).

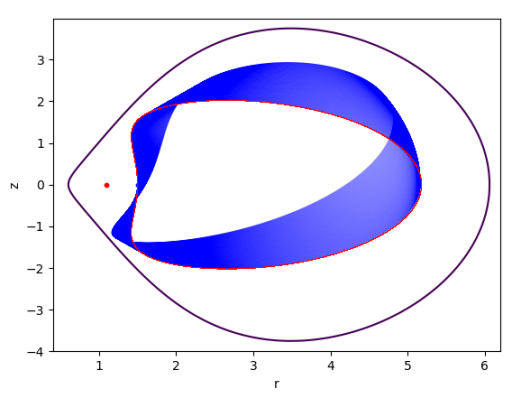

(a) CI: $(1.5,0,0,-0.74)$

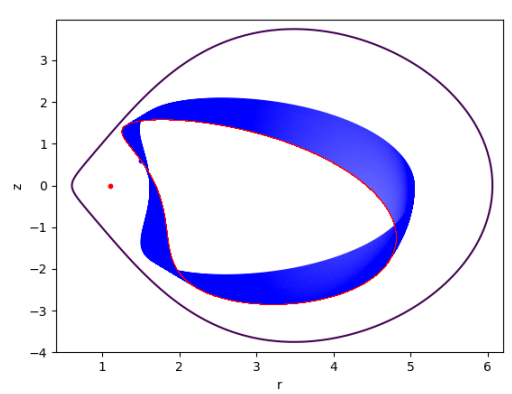

(d) CI: $(1.5,0.6,0.25,-0.62)$

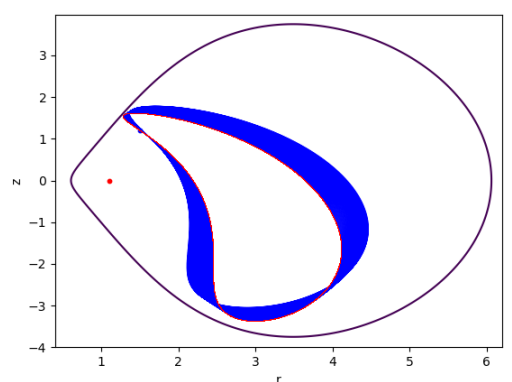

(g)

CI: $(1.5,1.2,0.31,-0.39)$

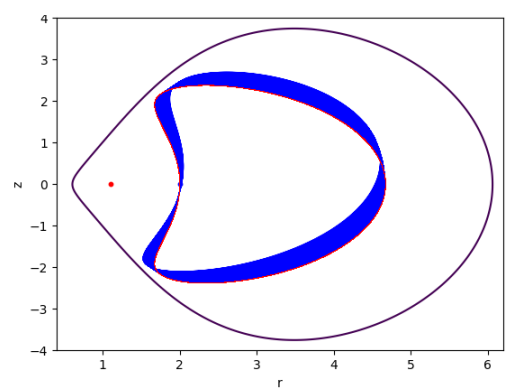

(b) CI: $(2.0,0,0,-0.65)$

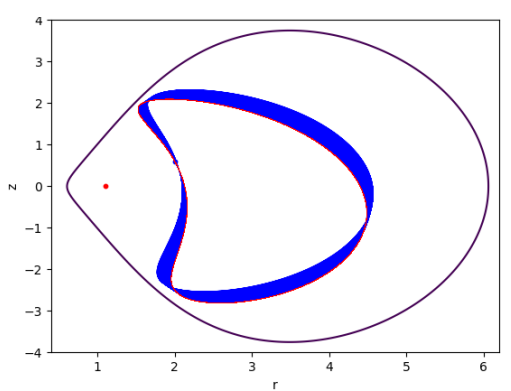

(e) CI: $(2.0,0.6,0.18,-0.59)$

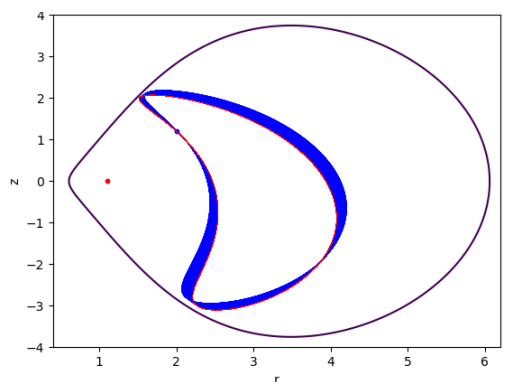

(h)

CI: $(2.0,1.2,0.27,-0.46)$

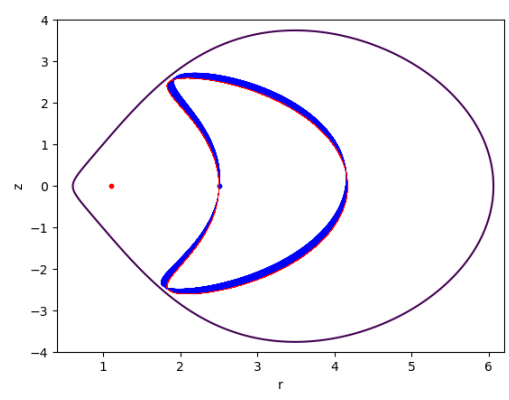

(c) CI: $(2.5,0,0,-0.57)$

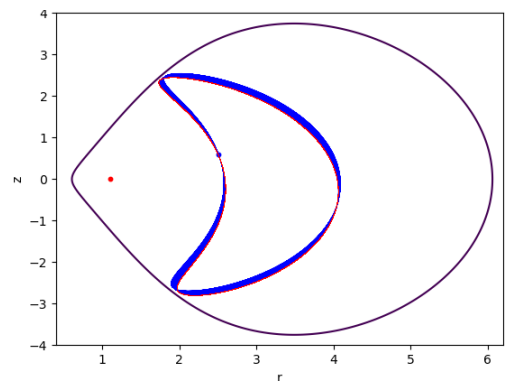

(f) CI: $(2.5,0.6,0.13,-0.53)$

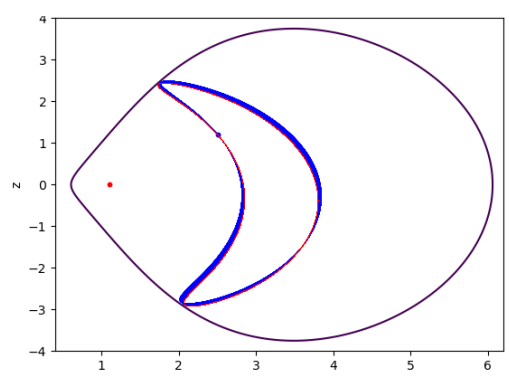

(i) CI: $(2.5,1.2,0.21,-0.45)$

Figura 1.3: Soluções numéricas no espaço de configurações $(r, z)$, em função da condição inicial $\mathrm{CI}=\left(r_{0}, z_{0}, p_{r, 0}, p_{z, 0}\right)$. Figuras na mesma linha correspondem a variações na condição inicial $r_{0}$, figuras na mesma coluna correspondem a variações na condição inicial $z_{0}$. A curva vermelha corresponde à solução obtida com as mesmas condições iniciais no caso esférico $(e=0)$, e a curva preta delineia o bordo da bacia permitida do potencial efetivo, caracterizada pela equação $\Phi_{\mathrm{eff}}(r, z)=E$. O ponto azul representa a condição inicial e o vermelho representa a posição $\left(r_{+}, 0\right)$ do minimo do potencial efetivo. Os valores dos demais parâmetros utilizados foram $E=-0.15, e=0.10$ e $l_{z}=1.05$. 


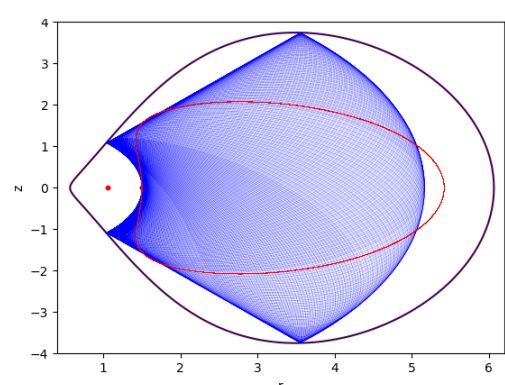

(a) CI: $(1.5,0,0,-0.74)$

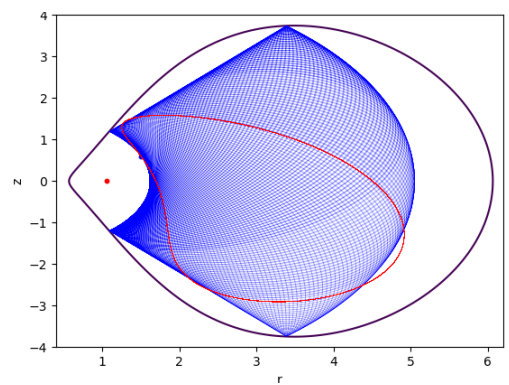

(d)

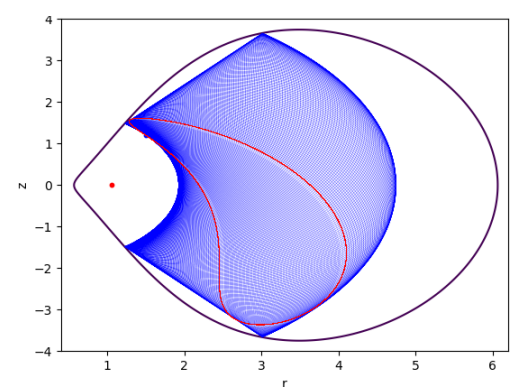

(g)

$$
\text { CI: }(1.5,1.2,0.31,-0.39)
$$

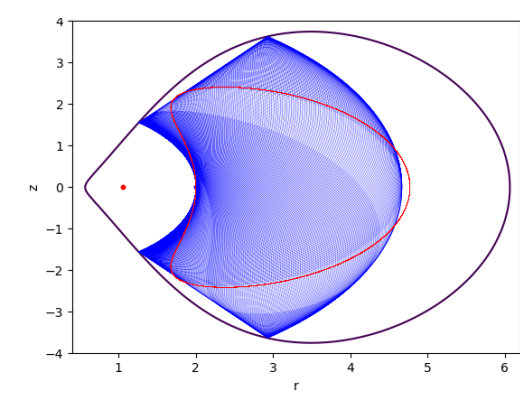

(b) CI: $(2.0,0,0,-0.65)$

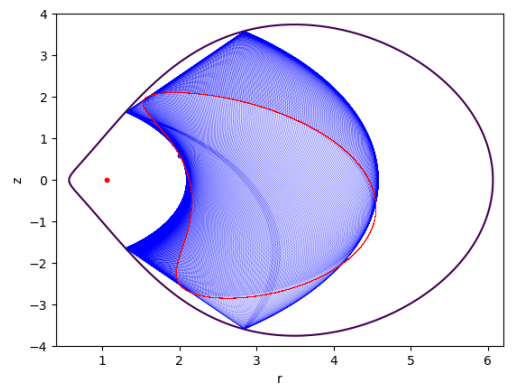

(e) CI: $(2.0,0.6,0.18,-0.59)$

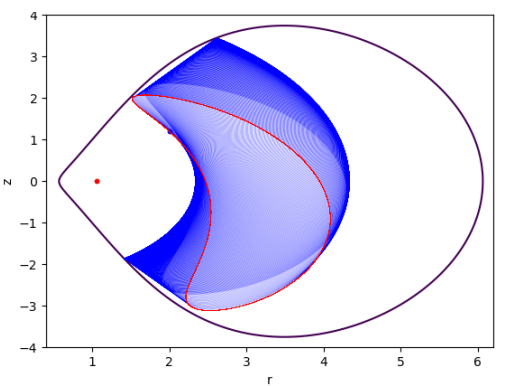

(h) CI: $(2.0,1.2,0.27,-0.46)$

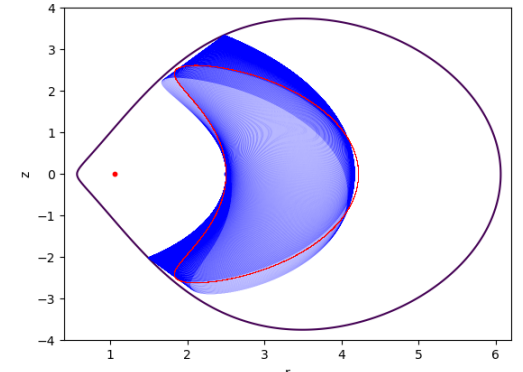

(c) CI: $(2.5,0,0,-0.57)$

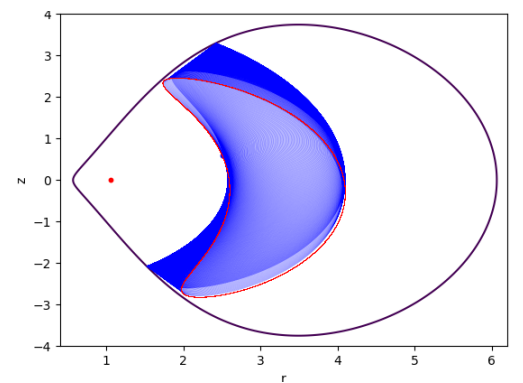

(f) CI: $(2.5,0.6,0.13,-0.54)$

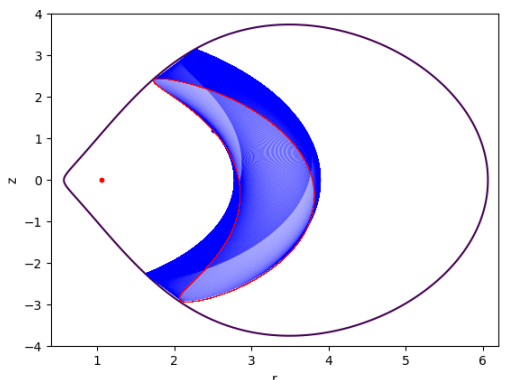

(i) CI: $(2.5,1.2,0.21,-0.45)$

Figura 1.4: Soluções numéricas no espaço de configurações $(r, z)$, em função da condição inicial $\mathrm{CI}=\left(r_{0}, z_{0}, p_{r, 0}, p_{z, 0}\right)$. Figuras na mesma linha correspondem a variaçôes na condição inicial $r_{0}$, figuras na mesma coluna correspondem a variações na condição inicial $z_{0}$. A curva vermelha corresponde à solução obtida com as mesmas condições iniciais no caso esférico $(e=0)$, e a curva preta delineia o bordo da bacia permitida do potencial efetivo, caracterizada pela equação $\Phi_{\mathrm{eff}}(r, z)=E$. O ponto azul representa a condição inicial e o vermelho representa a posição $\left(r_{+}, 0\right)$ do minimo do potencial efetivo. Os valores dos demais parâmetros utilizados foram $E=-0.15, e=0.43$ (o valor de Saturno) $e l_{z}=1.05$.

Como esperado, quando $e=0$, toda órbita é periódica, enquanto que se $e>0$, ela apresenta desvios da correspondente órbita periódica que se acumulam ao longo do tempo, mostrando uma tendência dela, no limite $t \rightarrow \infty$, "preencher" uma certa região no espaço de configurações. Estas regiões mostram uma interessante estrutura que, até agora, parece pouco compreendida.

Quanto à dependência das condições iniciais e dos parâmetros do problema, notamos o seguinte. Primeiro, variação da condição inicial $z_{0}$ não produz mudança significativa na imagem obtida. Segundo, variação da condição inicial $r_{0}$ também mostra o comportamento esperado: na medida em que $r_{0}$ aumenta, a contribuição do termo quadrupolo diminui e as órbitas perturbadas ficam mais próximas às órbitas periódicas correspondentes. E por fim, um aumento da elipsidade, como da Fig. 1.3 para a Fig. 1.4, permite demonstrar os efeitos dessa perturbação de maneira muito mais nítida.

Os resultados numéricos sustentam a conjectura de que o fecho de cada órbita perturbada, com energia $E$, ocupa apenas um subconjunto não trivial - com bordo composto pela união de curvas suaves - da correspondente bacia do potencial efetivo, $\left\{(r, z) \mid \Phi_{\text {eff }}(r, z) \leqslant E\right\}$. Encontrar equações que caracterizem estas curvas, em função das condições iniciais e dos parâmetros, permanece um problema em aberto. 
Outra questão interessante é estudar o comportamento do ângulo $\alpha$, introduzido na seção anterior, que caracteriza a inclinação da órbita em relação ao plano equatorial. A simulação numérica revela que $\alpha$ é uma função oscilatória do tempo:

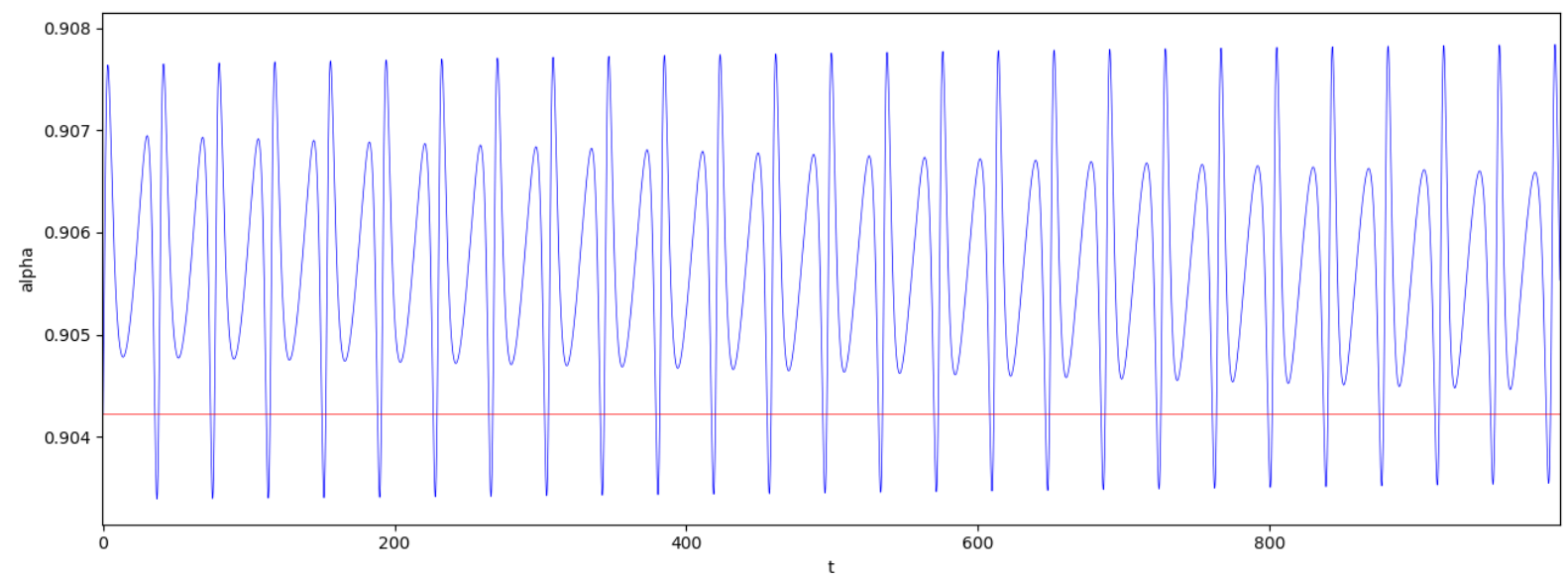

Figura 1.5: Diagrama representando o valor do ângulo $\alpha$ como função do tempo. A Linha azul corresponde ao caso perturbado, enquanto a vermelha ao caso esférico. Os parâmetros utilizados foram $E=-0.15$, $e=0.43, l_{z}=1.05$ com condições iniciais $(1.5,1.5,0.27,-0.27)$ e tempo final $t=1000$.

Em uma escala de tempo maior, a mesma utilizada nas Figuras 1.3 e 1.4 ( $\mathrm{t}=10000)$, evidenciam-se periodicidades adicionais, de frequência muito mais baixa, modulando a amplitude destas oscilações:

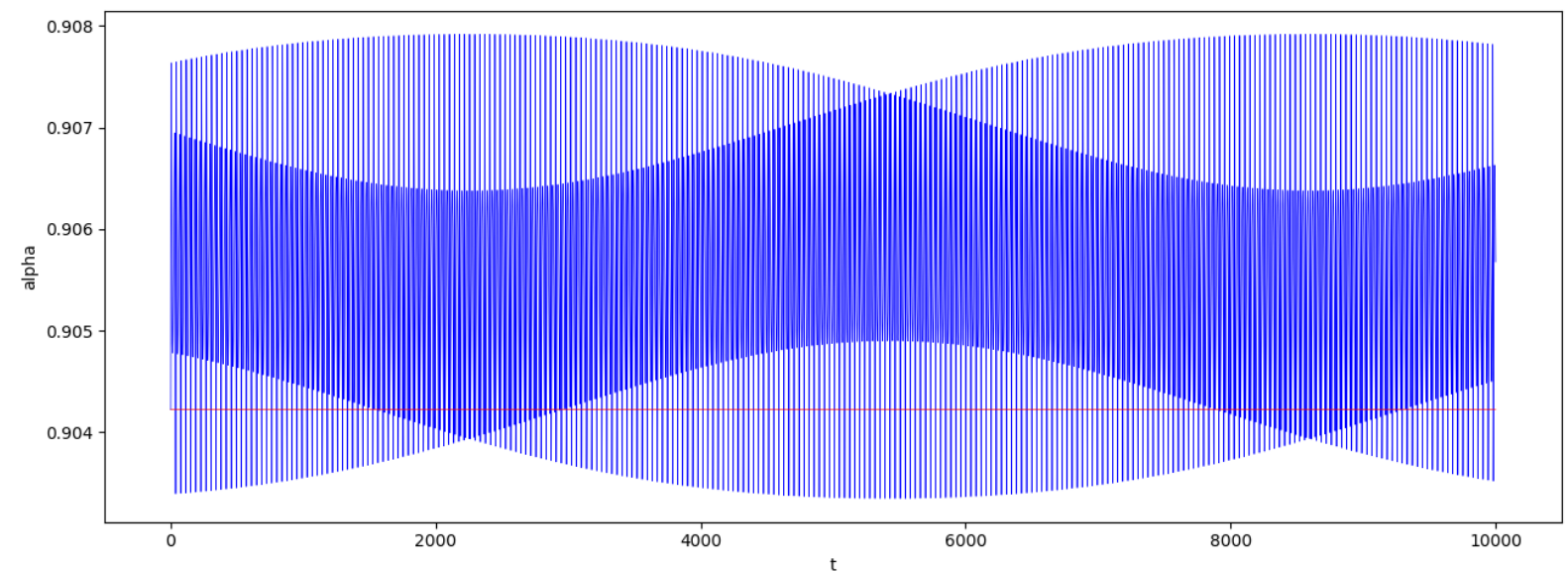

Figura 1.6: Diagrama representando o valor do ângulo a como função do tempo. A Linha azul corresponde ao caso perturbado, enquanto a vermelha ao caso esférico. Os parâmetros utilizados foram $E=-0.15$, $e=0.43, l_{z}=1.05$ com condições iniciais $(1.5,1.5,0.27,-0.27)$ e tempo final $t=10000$

Novamente, determinar as causas dessa estrutura temporal permanece um problema em aberto.

\subsection{Sobre o fenômeno de achatamento}

Tendo em vista os resultados obtidos nas seções anteriores, podemos voltar a contemplar a nossa questão original: existe a possibilidade de um termo de quadrupolo no potencial gravitacional contribuir ao fenômeno de achatamento e, assim, a formação de discos?

Claramente, pode-se dizer: por si só, não! No entanto, a introdução deste termo faz com que, aparentemente, a órbita - que antes era periódica e de medida nula no espaço de configurações $(r, z)$ - agora seja densa em um novo subconjunto da bacia do potencial efetivo que tem medida positiva. Sendo assim, considerando um cenário astronômico mais realista, onde estamos lidando não com uma única partícula orbitando a massa central e sim com uma "nuvem" de partículas, 
cada uma preenchendo seu respectivo subconjunto da bacia do potencial, é de se imaginar que colisões entre estas partículas ocorrerão com probabilidade muito maior do que na ausência da perturbação. Durante tais colisões, parte da energia cinética das partículas envolvidas é termalizada, inclusive sendo emitida na forma de radiação, fazendo com que caiam cada vez mais fundo no poço gravitacional e se acumulem perto do plano equatorial. Portanto, é neste sentido coletivo que o termo quadrupolo pode sim dar uma contribuição ao achatamento e a formação de discos em astronomia. 


\section{Capítulo 2}

\section{Órbitas especiais}

Como vimos no capítulo anterior, o conjunto $\Delta$ - correspondente ao plano equatorial - é invariante sobre ação do fluxo hamiltoniano. Ou seja, restrito a esse conjunto o sistema passa a ter apenas um grau de liberdade e é, portanto, completamente integrável. Além disso, vimos que existem dois pontos de equilíbrio, dados por $\mathbf{r}_{+}=\left(r_{+}, 0,0,0\right)$ e $\mathbf{r}_{-}=\left(r_{-}, 0,0,0\right)$, que correspondem, respectivamente, aos pontos de mínimo e máximo locais do potencial efetivo restrito a $\Delta$. Na figura abaixo vemos o retrato de fases para dois valores distintos de $l_{z}$, o primeiro corresponde ao caso representado anteriormente em Fig. 1.1(b), o segundo em Fig. 1.1(c):

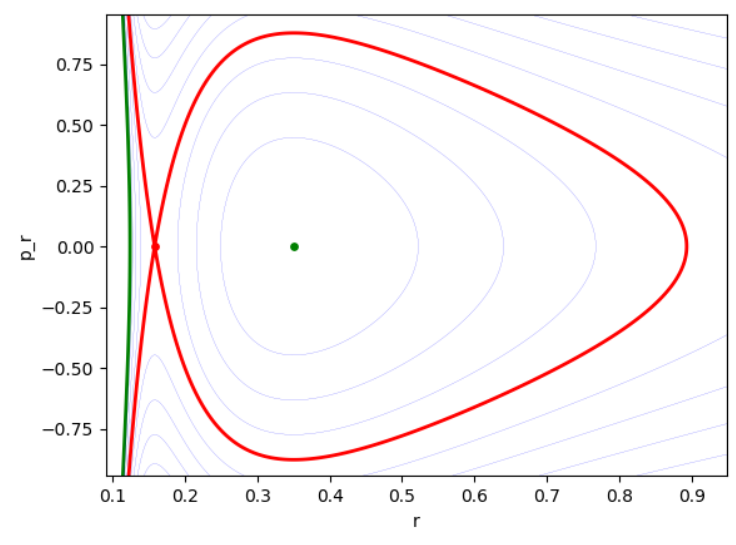

(a) $\quad l^{2}=7 e^{2} / 5$

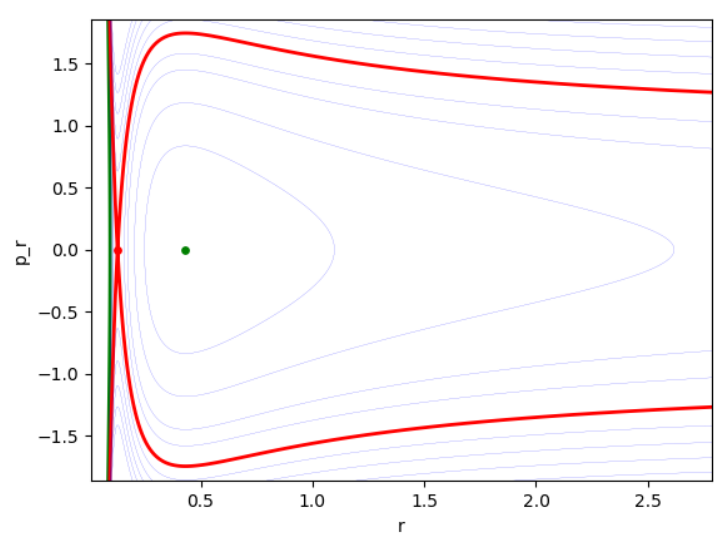

(b) $\quad l^{2}=8.5 e^{2} / 5$

Figura 2.1: O retrato de fases de soluções no plano equatorial ( $z=p_{z}=0$ para todo $\left.t \in \mathbb{R}\right)$. O ponto vermelho representa o equilíbrio $\mathbf{r}_{-}$, o ponto verde representa o equilíbrio $\mathbf{r}_{+} \cdot$ As curvas destacadas em vermelho são as órbitas com energia dadas por $\Phi_{\text {eff }}\left(r_{-}, 0\right)$ e em verde por $\Phi_{\text {eff }}\left(r_{+}, 0\right)$. As legendas de cada uma das subfiguras indicam os valores de $l^{2}$. A elipsidade utilizada foi a de Saturno, e $=0.43$.

Na Fig. 2.1 podemos identificar duas órbitas especiais que são os objetos de estudo deste capítulo. A primeira seção tem como objetivo obter uma solução aproximada em uma vizinhança da órbita representada pelo ponto verde, que nada mais é do que a solução trivial associada ao ponto estável. Vale destacar que nosso foco está apenas na situação de maior relevância física, o caso em que $r_{+}>1$. Por outro lado, na segunda seção estaremos interessados no comportamento dinâmico do sistema em uma vizinhança da órbita homoclínica (bi-assintótica) ao ponto de equilíbrio $\mathbf{r}_{-}$, destacada em vermelho no caso (a). 


\section{1 Órbita cirular e a aproximação epicíclica}

Deste ponto em diante, nos referimos a solução dada pelo ponto de equilíbrio $\mathbf{r}_{+}$como órbita circular. Explicitamente, a solução do problema original em três dimensões associado a tal órbita é $\Gamma_{\text {circ }}(t)=\left(r_{+}, 0, v t+\varphi_{0}\right)$, onde $v$ é uma constante dada por $v=l_{z} r_{+}^{-2}$. Uma primeira aproximação do potencial em uma vizinhança dessa órbita é completamente integrável e suas soluções são dadas por movimentos harmônicos simples. Essa aproximação é conhecida como aproximação epicíclica.

Considere $l_{z}^{2}$ satisfazendo a condição (1.74), de forma que $r_{+}>1$. Soluções próximas a $\mathbf{r}_{+}=\left(r_{+}, 0,0,0\right)$ podem ser aproximadas considerando a expansão de Taylor de $\Phi_{\text {eff }}(r, z)$ em torno de $\left(r_{+}, 0\right)$, ou seja, escrevemos:

$$
\begin{aligned}
\Phi_{\mathrm{eff}}(r, z)= & \Phi_{\mathrm{eff}}\left(r_{+}, 0\right)+\partial_{r} \Phi_{\mathrm{eff}}\left(r_{+}, 0\right)\left(r-r_{+}\right)+\partial_{z} \Phi_{\mathrm{eff}}\left(r_{+}, 0\right) z \\
& +\frac{1}{2} \partial_{r}^{2} \Phi_{\mathrm{eff}}\left(r_{+}, 0\right)\left(r-r_{+}\right)^{2}+\frac{1}{2} \partial_{z}^{2} \Phi_{\mathrm{eff}}\left(r_{+}, 0\right) z^{2} \\
& +\frac{1}{2} \partial_{r} \partial_{z} \Phi_{\mathrm{eff}}\left(r_{+}, 0\right)\left(r-r_{+}\right) z+\ldots
\end{aligned}
$$

Mas segue das equações (1.60), (1.61) e (1.66) que

$$
\partial_{r} \partial_{z} \Phi_{\text {eff }}\left(r_{ \pm}, 0\right)=\partial_{r} \Phi_{\text {eff }}\left(r_{ \pm}, 0\right)=\partial_{z} \Phi_{\text {eff }}\left(r_{ \pm}, 0\right)=0
$$

e assim, ignorando os termos de ordem superior, concluimos que em torno de $\left(r_{+}, 0\right)$ vale que

$$
\Phi_{\mathrm{eff}}(r, z) \approx \Phi_{\mathrm{eff}}\left(r_{+}, 0\right)+\partial_{r}^{2} \Phi_{\mathrm{eff}}\left(r_{+}, 0\right) \frac{\left(r-r_{+}\right)^{2}}{2}+\partial_{z}^{2} \Phi_{\mathrm{eff}}\left(r_{+}, 0\right) \frac{z^{2}}{2} .
$$

Com essa aproximação e definindo

$$
\omega^{2}=\partial_{r}^{2} \Phi_{\mathrm{eff}}\left(r_{+}, 0\right) \quad \text { e } \quad \nu^{2}=\partial_{z}^{2} \Phi_{\mathrm{eff}}\left(r_{+}, 0\right),
$$

reescrevemos (1.58) como

$$
\left\{\begin{array}{l}
\ddot{r}=-\omega^{2} r \\
\ddot{z}=-\nu^{2} z
\end{array} .\right.
$$

E como podemos ver em (1.71) e (1.70)

$$
\partial_{r}^{2} \Phi_{\mathrm{eff}}\left(r_{+}, 0\right)>0, \quad \partial_{z}^{2} \Phi_{\mathrm{eff}}\left(r_{+}, 0\right)>0,
$$

dessa forma, a solução do sistema (2.5) é simplesmente a composição de movimentos harmônicos independentes de frequência $\omega$ em $r$ e $\nu$ em $z$ centralizados em $\mathbf{r}_{+}$. Explicitamente

$$
\begin{aligned}
& r(t)=A \cos (\omega t+a)+r_{+} \stackrel{\text { def }}{=} \delta(t)+r_{+}, \\
& z(t)=B \cos (\nu t+b)
\end{aligned}
$$

onde $A, a, B, b$ são constantes que estarão definidas dadas as condições iniciais. Resta calcular $\varphi(t)$. Para isso, lembre que neste caso

$$
\dot{\varphi}=\frac{l_{z}}{r^{2}}=\frac{l_{z}}{r_{+}^{2}}\left(1+\frac{\delta(t)}{r_{+}}\right)^{-2}
$$

Mas próximo a $\mathbf{r}_{+}$(ou equivalentemente, próximo a $\delta=0$ ), vale que

$$
\left(1+\frac{\delta}{r_{+}}\right)^{-2} \approx\left(1-\frac{2 \delta}{r_{+}}\right)
$$

Assim, dada uma condição inicial $\mathbf{u}_{\mathbf{0}}=\left(r_{0}+r_{+}, p_{r, 0}, z_{0}, p_{z, 0}, \varphi_{0}, l_{z}\right)$ a solução aproximada de (1.43) 
próximo a $\mathbf{r}_{+}$é

$$
\begin{aligned}
& r(t)=-\left(r_{0}^{2}+\frac{p_{r, 0}^{2}}{\omega^{2}}\right)^{1 / 2} \cos \left(\omega t+\arccos \left(-r_{0}\left(r_{0}^{2}+\frac{p_{r, 0}^{2}}{\omega^{2}}\right)^{-1 / 2}\right)\right)+r_{+} \\
& z(t)=-\left(z_{0}^{2}+\frac{p_{z, 0}^{2}}{\nu^{2}}\right)^{1 / 2} \cos \left(\nu t+\arccos \left(-z_{0}\left(z_{0}^{2}+\frac{p_{z, 0}^{2}}{\nu^{2}}\right)^{-1 / 2}\right)\right) \\
& \varphi(t)=\frac{L_{z}}{r_{+}^{2}}\left(t+\frac{2}{\omega r_{+}}\left(r_{0}^{2}+\frac{p_{r, 0}^{2}}{\omega^{2}}\right)^{1 / 2} \sin \left(\omega t+\arccos \left(-r_{0}\left(r_{0}^{2}+\frac{p_{r, 0}^{2}}{\omega^{2}}\right)^{-1 / 2}\right)\right)+\varphi_{0}\right.
\end{aligned}
$$

Para analisar a fidelidade dessa aproximação, nas próximas subseções iremos integrar (1.58) numericamente para diferentes condições iniciais e comparar com as soluções analíticas da aproximação epicíclica obtidas.

\subsubsection{Variações nos momentos}

Em Fig.(2.2) podemos constatar o fato (nem um pouco surpreendente) que a aproximação deixa de ser eficiente quanto maior o valor de $p_{r, 0}$ e $p_{z, 0}$.

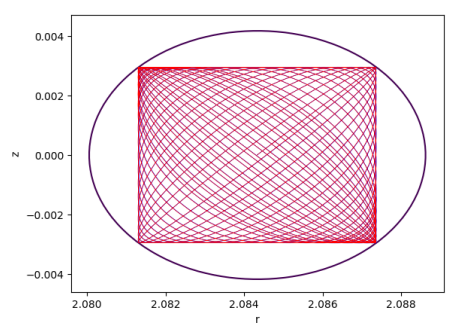

(a) $\quad p_{r, 0}=p_{z, 0}=10^{-3}$

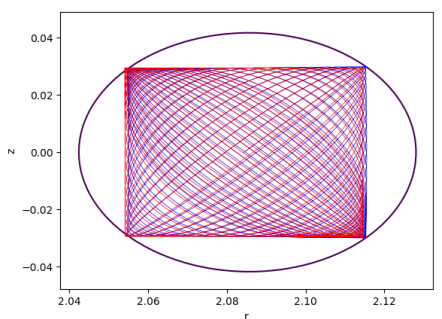

(b) $\quad p_{r, 0}=p_{z, 0}=10^{-2}$

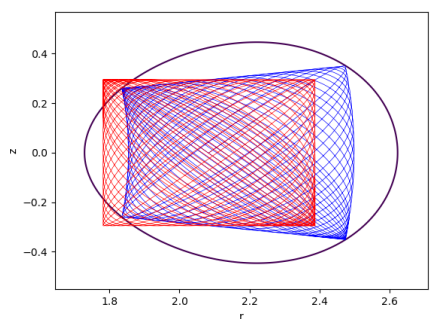

(c) $\quad p_{r, 0}=p_{z, 0}=10^{-1}$

Figura 2.2: A projeção no plano $(r, z)$ da solução aproximada (em vermelho) e da solução numérica (em azul) para condições iniciais $u_{0}=\left(r_{+}, p_{r, 0}, 0, p_{z, 0}\right)$. A curva preta representa a curva equipotencial. As legendas de cada uma das subfiguras indicam os valores de $p_{r, 0}$ e $p_{z, 0}$. As constantes utilizadas foram $e=0$ $e l^{2}=2\left(1+3 e^{2} / 10\right)$

Em (a) vemos uma grande conformidade entre a aproximação e a solução numérica. Em (b) a semelhança ainda é grande, porém, já podemos notar um leve deslocamento entre as soluções no eixo radial, levando a solução aproximada a cruzar a curva equipotencial. Finalmente, em (c), ficam evidentes as diferenças entre a aproximação e a solução numérica; a aproximação tem amplitude do movimento em $z$ constante, enquanto, na solução numérica, tal amplitude é diretamente proporcional ao valor de $r$. 


\subsubsection{Variações em $r$}

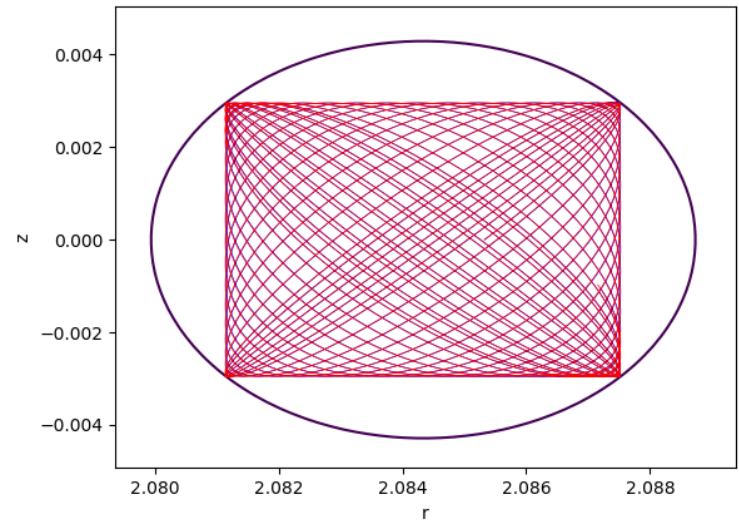

(a) $\quad r_{0}=10^{-3}$

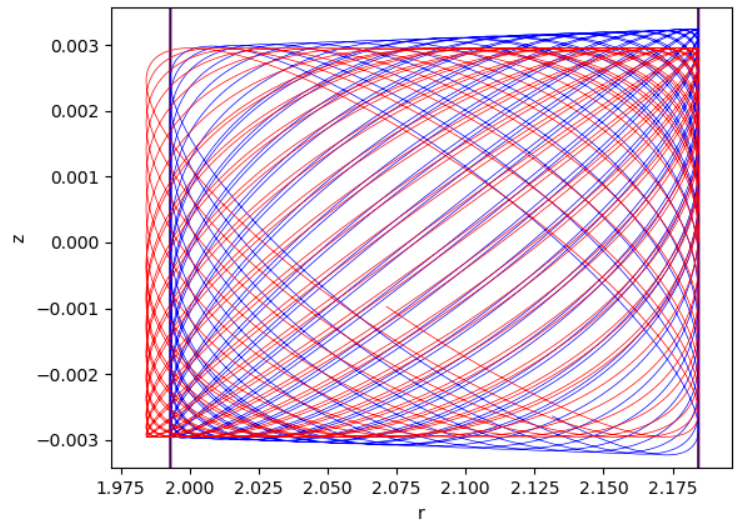

(c) $\quad r_{0}=10^{-1}$

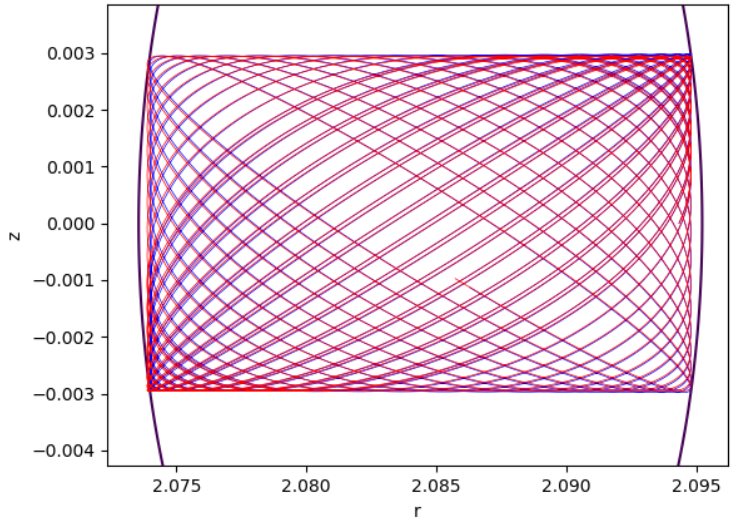

(b) $\quad r_{0}=10^{-2}$

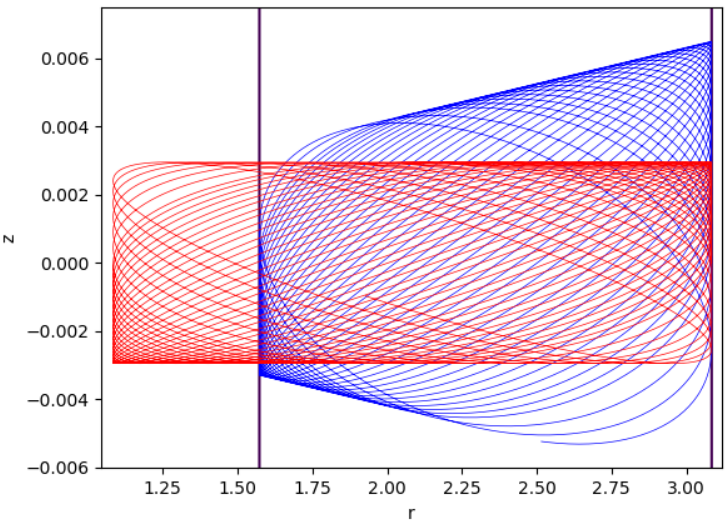

(d) $\quad r_{0}=1$

Figura 2.3: A projeção no plano $(r, z)$ da solução aproximada (em vermelho) e da solução numérica (em azul) para condições iniciais $u_{0}=\left(r_{+}+r_{0}, 10^{-2}, 0,10^{-2}\right)$. A curva preta representa a curva equipotencial. As legendas de cada uma das subfiguras indicam os valores de $r_{0}$. As constantes utilizadas foram $e=0$ e $l^{2}=2\left(1+3 e^{2} / 10\right)$.

Em (2.3) mantivemos os momentos da ordem de $10^{-3}$ - com os quais obtivemos bons resultados na subseção anterior - e alteramos o valor da variável $r$ das condições iniciais.

O resultado é similar ao obtido anteriormente, variando-se os momentos. Em (a) e (b) a aproximação é bastante satisfatória; em (c) ainda é possível notar grande semelhança entre aproximação e solução numérica (embora, neste caso, a aproximação escape consideravelmente da curva equipotencial), porém, em (d), a discrepância entre as duas fica evidente. 


\subsubsection{Variações em $z$}

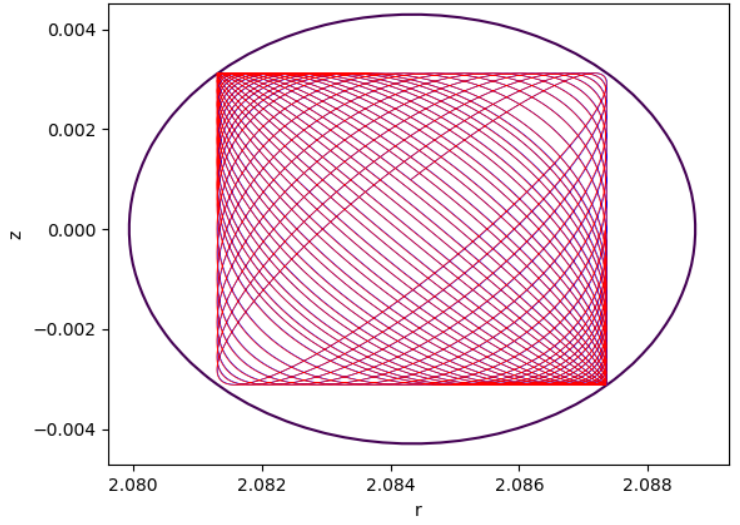

(a) $z_{0}=10^{-2}$

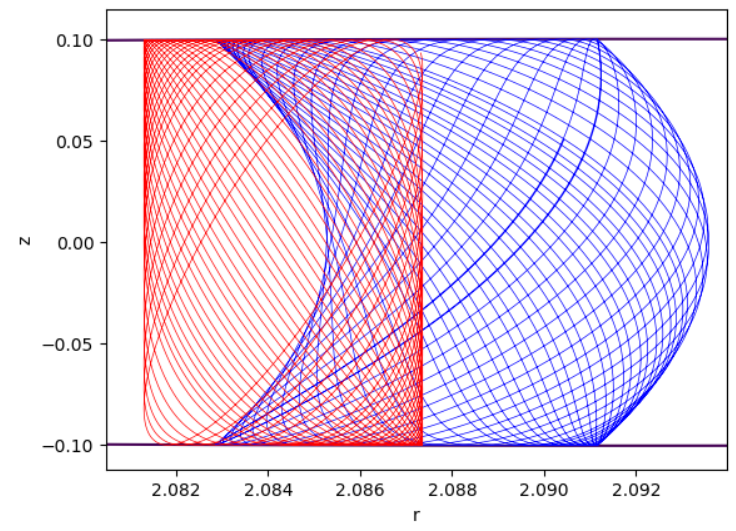

(c) $\quad z_{0}=10^{-1}$

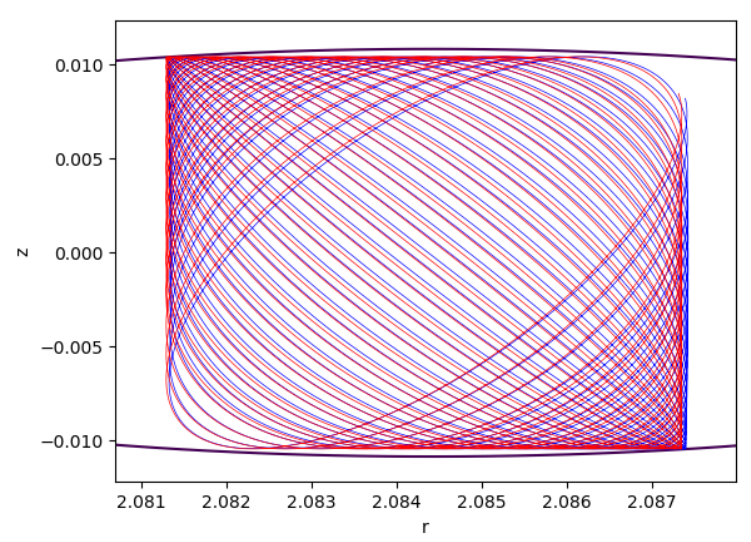

(b) $\quad z_{0}=10^{-2}$

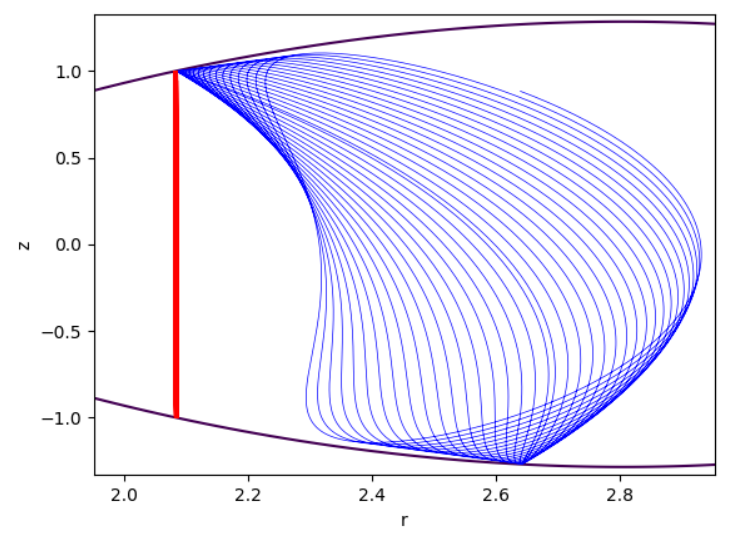

(d) $\quad z_{0}=1$

Figura 2.4: A projeção no plano $(r, z)$ da solução aproximada (em vermelho) e da solução numérica (em azul) para condições iniciais $u_{0}=\left(r_{+}, 10^{-2}, z_{0}, 10^{-2}\right)$. A curva preta representa a curva equipotencial. As legendas de cada uma das subfiguras indicam os valores de $z_{0}$. As constantes utilizadas foram $e=0$ e $l^{2}=2\left(1+3 e^{2} / 10\right)$.

Assim como na subseção anterior, mantivemos os momentos da ordem de $10^{-3}$. Neste caso, porém, pudemos notar uma sensibilidade muito maior com relação a mudanças na variável $z$, o que fica evidente em (c). Na subseção anterior, a variação da ordem de $10^{-1}$ ainda resultou em uma aproximação satisfatória, o que não é o caso aqui.

Com isso, conjectura-se que a aproximação epicíclica para o potencial discutido neste trabalho seja suficientemente eficiente apenas em vizinhanças da ordem de $10^{-2}$ da órbita circular no retrato de fases, ou equivalentemente, $1 \%$ do raio equatorial do esferoide.

Também vale destacar que não obtivemos nenhuma alteração significativa ao variar o valor da constante $e$, associada à elipsidade do esferoide. 


\section{2 Órbita homoclínica}

Na seção anterior, utilizamos uma aproximação para estudar o comportamento do sistema em uma região próxima a órbita circular. Agora, estamos interessados no ponto fixo instável do caso em que $6 e^{2} / 5<l_{z}^{4}<8 e^{2} / 5$. Como já sabemos, com essa restrição sobre $l_{z}$, ambos os equilíbrios estão localizados no interior do esferoide do modelo inicial e sendo assim, pode-se questionar a relevância física do resultado a seguir.

Veremos que o equilíbrio instável é do tipo centro-sela (possui um par de autovalores associados do tipo imaginário puro $\pm \omega i$ e outro par do tipo real puro $\pm \nu$ ), que existe uma órbita $\Gamma_{h}$ homoclínica (bi-assintótica) a tal ponto, com energia $h$ e uma família de soluções parametrizadas pelo seu nível energia, que se acumulam em $\Gamma_{h}$. O conjunto composto pela união de $\Gamma_{h}$ e este ponto fixo será chamado de "laço homoclínico".

Tais laços homoclínicos já foram estudados extensivamente na literatura, no contexto de sistemas hamiltonianos com dois graus de liberdade. Podemos citar, por exemplo, os trabalhos de Mielke et al. [MHO92], Lerman [Ler91], Letelier et al. [LRCLS11] e Depetri e Saa[DS15], que apresentam resultados relacionados ao comportamento caótico de soluções próximas ao laço. Estamos interessados, porém, na dinâmica do sistema.

Por isso, utilizaremos um método descrito por Ragazzo em [Rag97b] que consiste em estudar o problema através da função $f$ de primeiro retorno de Poincaré definida em uma seção $\Sigma$, transversal à família de soluções citadas acima. Em particular, estamos interessados na estabilidade ${ }^{1}$ do laço sob ação do fluxo hamiltoniano.

A conservação de energia nos permite expressar $f$ como uma família a um parâmetro de funções $f_{E}: \Sigma_{E} \rightarrow \Sigma_{E}$, onde $\Sigma_{E}$ é a restrição de $\Sigma$ ao nível de energia E.

Veremos que existe um sistema de coordenadas natural em $\Sigma_{E}$, tal que a origem $\mathbf{0}$ representa a interseção de $\Sigma$ com a órbita $\Gamma_{E} \subset \Delta$. A diferencial de $f_{E}$ em $\mathbf{0}$, quando definida, determina a estabilidade de $\mathbf{0}$ sob a ação de tal mapa, que por sua vez determina a estabilidade de $\Gamma_{E}$ pelo fluxo do sistema. No caso da órbita homoclínica, $f_{h}$ não é diferenciável na origem, nem sequer contínua. No entanto, Lerman e Mielke et al demonstraram que para valores de $E$ suficientemente próximos a $h$ e $\mathbf{y} \in \Sigma_{E}$ suficientemente próximo à origem, a dinâmica em $\Sigma_{E}$ por $f_{E}$ pode ser aproximada pela transformação continua:

$$
F_{E}(\mathbf{y})=A R\left(c-\gamma \ln \left|\frac{1}{2} \omega\|\mathbf{y}\|^{2}-(E-h)\right|\right) \mathbf{y},
$$

onde

$$
\begin{gathered}
\|\mathbf{y}\|>(2(E-h) / \omega)^{1 / 2} \text { para } E>h, \\
\gamma=\omega / \nu, \\
R(v) \stackrel{\text { def }}{=}\left(\begin{array}{cc}
\cos v & -\sin v \\
\sin v & \cos v
\end{array}\right), \quad A \stackrel{\text { def }}{=}\left(\begin{array}{cc}
\alpha & 0 \\
0 & 1 / \alpha
\end{array}\right) .
\end{gathered}
$$

e $c$ é uma constante. No caso em que $E=h$, a origem será, por definição, um ponto fixo de $F_{h}$.

Adotando o sistema de coordenadas esféricas, a constante $\alpha$ será determinada pela equação de Schrödinger unidimensional e homogênea no tempo, associada à linearização das equações de movimento das variáveis $\left(\theta, p_{\theta}\right)$ em $\Gamma_{h}$.

Assim, iremos estudar a dinâmica de $\Gamma_{h}$ e de soluções em sua vizinhança analisando a estabilidade da origem de $\Sigma_{E}$ sob ação do mapa $F_{E}$ e seus parâmetros $\alpha$ e $\gamma$.

\footnotetext{
${ }^{1}$ Diremos que um conjunto $X$ é estável sob ação de um fluxo $\Phi$ se, para qualquer vizinhança $U$ de $X$, existir uma vizinhança $V \subset U$ de $\mathrm{X}$ de maneira que toda órbita $\phi \operatorname{com} \phi(0) \in V$ é tal que $\phi(t) \in U$, para todo $t>0$.
} 


\subsubsection{O cálculo de $\alpha$}

Os primeiros passos são calcular os autovalores e a solução explícita de $\Gamma_{h}$. Para isso, considere o potencial em coordenadas esféricas:

$$
\Phi(R, \theta)=-\frac{1}{R}-\frac{e^{2}\left(1-3 \cos ^{2} \theta\right)}{10 R^{3}}
$$

com Hamiltoniana $\mathcal{H}$ associado

$$
\mathcal{H}=\frac{1}{2}\left(p_{R}^{2}+\frac{p_{\varphi}^{2}}{R^{2} \sin ^{2} \theta}+\frac{p_{\theta}^{2}}{R^{2}}\right)+\Phi(R, \theta) .
$$

Como já sabemos, $p_{\varphi}$ é uma integral primeira, assim, definindo $l_{z}=p_{\varphi}$, reescrevemos

$$
\begin{aligned}
\mathcal{H} & =\frac{1}{2}\left(p_{R}^{2}+\frac{p_{\theta}^{2}}{R^{2}}\right)+\frac{l_{z}^{2}}{2 R^{2} \sin ^{2} \theta}-\frac{1}{R}-\frac{e^{2}\left(1-3 \cos ^{2} \theta\right)}{10 R^{3}} \\
& =\frac{1}{2}\left(p_{R}^{2}+\frac{p_{\theta}^{2}}{R^{2}}\right)+\Phi_{l_{z}}(R, \theta),
\end{aligned}
$$

com equações de movimento dadas por

$$
\begin{aligned}
\dot{R} & =p_{R} \\
\dot{\theta} & =\frac{p_{\theta}}{R^{2}} \\
\dot{p_{R}} & =\frac{p_{\theta}^{2}}{R^{3}}+\frac{l_{z}^{2}}{R^{3} \sin ^{2} \theta}-\frac{1}{R^{2}}-\frac{3 e^{2}\left(1-3 \cos ^{2} \theta\right)}{10 R^{4}} \\
\dot{p_{\theta}} & =\frac{l_{z}^{2} \cos \theta}{R^{2} \sin ^{3} \theta}+\frac{3 e^{2} \cos \theta \sin \theta}{5 R^{3}}
\end{aligned}
$$

Note que em $\Delta=\left\{\left(R, \pi / 2, p_{R}, 0\right) \mid R \in \mathbb{R}_{+}, p_{R} \in \mathbb{R}\right\}$ vale

$$
\ddot{R}=-\frac{1}{R^{2}}+\frac{l_{z}^{2}}{R^{3}}-\frac{3 e^{2}}{10 R^{4}} .
$$

Os pontos fixos de (2.18) são os mesmos encontrados anteriormente; ou seja

$$
R_{ \pm}=\frac{1}{2}\left(l_{z}^{2} \pm \sqrt{l_{z}^{4}-6 e^{2} / 5}\right)
$$

Como estamos interessados no caso em que $6 e^{2} / 5<l_{z}^{4}<8 e^{2} / 5$, o ponto de equilíbrio $\mathbf{R}_{-}=\left(R_{-}, \pi / 2,0,0\right)$ possui autovalores

$$
\pm \nu= \pm \sqrt{\frac{2}{R_{-}^{3}}-\frac{3 l_{z}^{2}}{R_{-}^{4}}+\frac{6 e^{2}}{5 R_{-}^{5}}} \quad \text { e } \quad \pm i \omega= \pm i \sqrt{\frac{l_{z}^{2}}{R_{-}^{4}}+\frac{3 e^{2}}{5 R_{-}^{5}}} .
$$

O radicando de $\omega$ é estritamente positivo, portanto esses autovalores são puramente imaginários. Por outro lado, desde que $l_{z}^{4}>6 e^{2} / 5$, o radicando de $\nu$ será positivo e, portanto, os autovalores serão puramente reais. Para uso futuro definimos

$$
\gamma=\frac{\omega}{\nu}=\sqrt{\frac{l_{z}^{2} R_{-}+3 e^{2} / 5}{2 R_{-}^{2}-3 l_{z}^{2} R_{-}+6 e^{2} / 5}}
$$


Note que o denominador é equivalente a

$$
\begin{aligned}
2 R_{-}^{2}-3 l_{z}^{2} R_{-}+6 e^{2} / 5= & 2\left(l_{z}^{2}-\sqrt{l_{z}^{4}-6 e^{2} / 5}\right)^{2} \\
& -3 l_{z}^{2}\left(l_{z}^{2}-\sqrt{l_{z}^{4}-6 e^{2} / 5}\right)+6 e^{2} / 5 \\
= & -l_{z}^{2} R_{-}+3 e^{2} / 5
\end{aligned}
$$

de onde segue que

$$
\gamma=\sqrt{\frac{l_{z}^{2} R_{-}+3 e^{2} / 5}{-l_{z}^{2} R_{-}+3 e^{2} / 5}}
$$

Nessas condições, $\mathbf{R}_{-}$é um equilíbrio do tipo centro-sela e definindo $h \stackrel{\text { def }}{=} \Phi_{l_{z}}\left(R_{-}, \pi / 2\right)$, existe uma órbita $\Gamma_{h}$ em $\Delta$, relativa a esse nível de energia, que é homoclínica a $\mathbf{R}_{-}$. De fato, a componente radial de $\Gamma_{h}$ é dada pela solução da seguinte EDO de primeira ordem

$$
\begin{aligned}
\frac{d R}{d t} & =\sqrt{2} \sqrt{h-\Phi_{l_{z}}(R, \pi / 2)} \\
& =\sqrt{2} \sqrt{h+\frac{1}{R}-\frac{l_{z}^{2}}{2 R^{2}}+\frac{e^{2}}{10 R^{3}}} \\
& =\sqrt{2} \sqrt{\frac{|h|}{R^{3}}\left(-R^{3}+\frac{1 R^{2}}{|h|}-\frac{l_{z}^{2} R}{2|h|}+\frac{e^{2}}{10|h|}\right)} .
\end{aligned}
$$

Sabemos que $\Phi_{l_{z}}(R, \pi / 2)=h$ tem três soluções: $R_{-}$com multiplicidade 2 e um outro valor que definimos como $\bar{R}$. Portanto, o termo entre parenteses da equação acima deve se decompor como

$$
-R^{3}+\frac{R^{2}}{|h|}-\frac{l_{z}^{2} R}{2|h|}+\frac{e^{2}}{10|h|}=\left(R-R_{-}\right)^{2}(\bar{R}-R) .
$$

Por outro lado

$$
\left(R-R_{-}\right)^{2}(\bar{R}-R)=-R^{3}+R^{2}\left(\bar{R}+2 R_{-}\right)-R\left(R_{-}^{2}+2 \bar{R} R_{-}\right)+\bar{R} R_{-}^{2},
$$

o que motiva as seguintes relações

$$
\bar{R}+2 R_{-}=\frac{1}{|h|}, \quad R_{-}^{2}+2 \bar{R} R_{-}=\frac{l_{z}^{2}}{2|h|}, \quad \bar{R} R_{-}^{2}=\frac{e^{2}}{10|h|},
$$

que serão utilizadas com frequência ao longo do trabalho. Note, por exemplo que:

$$
\gamma=\sqrt{\frac{l_{z}^{2} R_{-}+3 e^{2} / 5}{-l_{z}^{2} R_{-}+3 e^{2} / 5}}=\sqrt{-\frac{\frac{2 l_{z}^{2} R_{-}+12 e^{2} / 10}{\left(\bar{R}-R_{-}\right)|h|}}{\frac{2 l_{z}^{2} R_{-}-12 e^{2} / 10}{\left(\bar{R}-R_{-}\right)|h|}}},
$$


e utilizando (2.27), obtemos que o denominador do radicando é dado por

$$
\begin{aligned}
\frac{2 l_{z}^{2} R_{-}-12 e^{2} / 10}{\left(\bar{R}-R_{-}\right)|h|} & =\frac{4 l_{z}^{2} R_{-}}{2\left(\bar{R}-R_{-}\right)|h|}-\frac{12 e^{2}}{10\left(\bar{R}-R_{-}\right)|h|} \\
& =\frac{4 R_{-}\left(R_{-}^{2}+2 \bar{R} R_{-}\right)}{\bar{R}-R_{-}}-\frac{12 R^{2} \bar{R}}{\bar{R}-R_{-}} \\
& =\frac{4 R^{2}\left(R_{-}-\bar{R}\right)}{\left(\bar{R}-R_{-}\right)}=-4 R^{2}
\end{aligned}
$$

e, finalmente,

$$
\gamma=\frac{1}{2} \sqrt{\frac{2 l_{z}^{2} R_{-}+6 e^{2} / 5}{R_{-}^{2}\left(\bar{R}-R_{-}\right)|h|}} .
$$

A utilidade prática de tal simplificação ficará clara no final da seção.

Agora, para encontrar explicitamente a solução $\Gamma_{h}$, considere a equação

$$
\frac{d \varrho}{d s}=\sqrt{2|h|} \sqrt{-r^{4}+\frac{r^{3}}{|h|}-\frac{l_{z}^{2} r^{2}}{2|h|}+\frac{e^{2}}{10|h|}}
$$

Veremos que $\varrho$ nada mais é do que $R$ quando considerado como função da nova variável temporal $s$, que será definida futuramente. Como (2.31) é separável, segue que

$$
\int_{\bar{R}}^{R(s)} \frac{d \xi}{\sqrt{\xi\left(\xi-R_{-}\right)^{2}(\bar{R}-\xi)}}=\sqrt{2|h|} .
$$

Fazendo $\xi=\bar{R}\left(1-x^{2}\right)$, o lado esquerdo se reescreve como

$$
\begin{aligned}
\int \frac{d \xi}{\sqrt{\xi\left(\xi-R_{-}\right)(\bar{R}-\xi)}} & =\int \frac{d \xi}{\xi\left(\xi-R_{-}\right) \sqrt{\frac{\bar{R}}{\xi}-1}} \\
& =\int d x \frac{-2 \bar{R} x}{\bar{R}\left(1-x^{2}\right)\left(\bar{R}\left(1-x^{2}\right)-R_{-}\right) \sqrt{\frac{1}{1-x^{2}}-1}} \\
& =\int \frac{-2 d x}{\bar{R}\left(1-x^{2}\right)\left(\bar{R}\left(1-x^{2}\right)-R_{-}\right) \sqrt{1-x^{2}}} \\
& =-\frac{2}{\bar{R}} \int \frac{d x}{\left(c^{2}-x^{2}\right) \sqrt{1-x^{2}}}
\end{aligned}
$$

onde $c^{2}=\left(\bar{R}-R_{-}\right) \bar{R}^{-1}$. Tomando a mudança de variáveis dada por $x=2 y\left(1+y^{2}\right)^{-1}$, segue que

$$
-\frac{2}{\bar{R}} \int \frac{d x}{\left(c^{2}-x^{2}\right) \sqrt{1-x^{2}}}=-\frac{4}{c^{2} \bar{R}} \int d y \frac{\left(1+y^{2}\right)}{y^{4}+\left(2-\frac{4}{c^{2}}\right) y^{2}+1} .
$$

Definimos

$$
S_{1}^{2}=\frac{-c^{2}-2 \sqrt{1-c^{2}}+2}{c^{2}} \quad \text { e } \quad S_{2}^{2}=\frac{-c^{2}+2 \sqrt{1-c^{2}}+2}{c^{2}}
$$


Dessa forma $S_{1}$ e $S_{2}$ satisfazem

$$
\begin{aligned}
S_{1}^{2} S_{2}^{2} & =\frac{1}{c^{4}}\left(\left(2-c^{2}\right)+2 \sqrt{1-c^{2}}\right)\left(\left(2-c^{2}\right)-2 \sqrt{1-c^{2}}\right) \\
& =\frac{1}{c^{4}}\left(\left(2-c^{2}\right)^{2}-4\left(1-c^{2}\right)\right) \\
& =1
\end{aligned}
$$

e, além disso,

$$
\begin{aligned}
\left(S_{1}-S_{2}\right)^{2} & =S_{1}^{2}+S_{2}^{2}-2 S_{1} S_{2} \\
& =\frac{4\left(1-c^{2}\right)}{c^{2}} \\
& =\frac{4 R_{-}}{\bar{R}-R_{-}}
\end{aligned}
$$

assim

$$
-\frac{4}{c^{2} \bar{R}} \int d y \frac{\left(1+y^{2}\right)}{y^{4}+\left(2-\frac{4}{c^{2}}\right) y^{2}+1}=-\frac{4}{c^{2} \bar{R}} \int d y \frac{S_{1} S_{2}+y^{2}}{\left(S_{1}^{2}-y^{2}\right)\left(S_{2}^{2}-y^{2}\right)}
$$

A integral do lado direito tem primitiva

$$
\log \frac{\left(y-S_{1}\right)\left(y+S_{2}\right)}{\left(y+S_{1}\right)\left(y-S_{2}\right)},
$$

que satisfaz

$$
\begin{aligned}
\log \left(\frac{\left(y-S_{1}\right)\left(y+S_{2}\right)}{\left(y+S_{1}\right)\left(y-S_{2}\right)}\right) & =\log \left(\frac{y^{2}+\left(S_{2}-S_{1}\right) y-S_{1} S_{2}}{y^{2}-\left(S_{2}-S_{1}\right) y-S_{1} S_{2}}\right) \\
& =\log \left(\frac{\frac{y^{2}-1}{\left(S_{2}-S_{1}\right) y}+1}{\frac{y^{2}-1}{\left(S_{2}-S_{1}\right) y}-1}\right) \\
& =2 \operatorname{arctanh}\left(\frac{y^{2}-1}{\left(S_{2}-S_{1}\right) y}\right)
\end{aligned}
$$

Portanto, (2.32) é equivalente a

$$
\frac{y^{2}-1}{\left(S_{2}-S_{1}\right) y}=\tanh (\zeta s),
$$

onde

$$
\zeta=\sqrt{\frac{R_{-}\left(\bar{R}-R_{-}\right)|h|}{2}} .
$$

Seja $k=\left(S_{2}-S_{1}\right) \tanh (\zeta s)$, então (2.41) é equivalente a

$$
y^{2}-k y-1=0
$$

e portanto

$$
y=\frac{k \pm \sqrt{k^{2}+4}}{2} .
$$


Como, pelas mudanças de variáveis,

$$
\varrho=\bar{R} \frac{\left(1-y^{2}\right)^{2}}{\left(1+y^{2}\right)^{2}}
$$

onde

$$
y^{2}-1=\frac{k^{2} \pm k \sqrt{k^{2}+4}}{2} \quad \text { e } \quad y^{2}+1=\frac{k^{2} \pm k \sqrt{k^{2}+4}+4}{2}
$$

segue que

$$
\begin{aligned}
& \varrho(s)=\bar{R} \frac{\left(1-y^{2}\right)^{2}}{\left(1+y^{2}\right)^{2}} \\
& =\bar{R} \frac{k^{2}}{k^{2}+4} \\
& =\frac{\bar{R}\left(S_{1}-S_{2}\right)^{2} \operatorname{coth}^{2}(\zeta s)}{\left(S_{1}-S_{2}\right)^{2} \operatorname{coth}^{2}(\zeta s)+4} \\
& =\frac{\frac{\bar{R} R_{-}}{\bar{R}-R_{-}} \operatorname{coth}^{2}(\zeta s)}{\frac{R_{-}}{\bar{R}-R_{-}} \operatorname{coth}^{2}(\zeta s)+\operatorname{coth}^{2}(\zeta s)-\operatorname{csch}^{2}(\zeta s)} \\
& =\frac{\bar{R} R_{-} \operatorname{coth}^{2}(\zeta s)}{\bar{R} \operatorname{coth}^{2}(\zeta s)-\left(\bar{R}-R_{-}\right) \operatorname{csch}^{2}(\zeta s)} \\
& =\frac{\bar{R} \cosh ^{2}(\zeta s)}{1+\frac{\bar{R}}{R_{-}}\left(\cosh ^{2}(\zeta s)-1\right)} \\
& =\frac{\bar{R} \cosh ^{2}(\zeta s)}{1+\frac{\bar{R}}{R_{-}} \sinh ^{2}(\zeta s)} \text {. }
\end{aligned}
$$

Finalmente, definindo

$$
t=t(s)=\int_{0}^{s} d \tau R^{2}(\tau)
$$

obtemos a solução de (2.24) como

$$
R(t)=\varrho(s(t))
$$

onde $s(t)$ é a inversa de (2.48).

Podemos então calcular a constante $\alpha$. Para isso linearizamos as equações de (2.17) referentes as coordenadas $\left(\theta, p_{\theta}\right)$ em $\Gamma_{h}$. Explicitamente, estamos interessados no seguinte sistema

$$
\left(\begin{array}{c}
\dot{\Theta} \\
\dot{P_{\Theta}}
\end{array}\right)=\left(\begin{array}{ll}
\frac{\partial \dot{\theta}}{\partial \theta}\left(\Gamma_{h}\right) & \frac{\partial \dot{\theta}}{\partial p_{\theta}}\left(\Gamma_{h}\right) \\
\frac{\partial \dot{p_{\theta}}}{\partial \theta}\left(\Gamma_{h}\right) & \frac{\partial \dot{p_{\theta}}}{\partial p_{\theta}}\left(\Gamma_{h}\right)
\end{array}\right)\left(\begin{array}{c}
\Theta \\
P_{\Theta}
\end{array}\right)
$$

E como

$$
\begin{gathered}
\frac{\partial \dot{\theta}}{\partial \theta}=\frac{\partial \dot{p_{\theta}}}{\partial p_{\theta}}=0, \\
\frac{\partial \dot{\theta}}{\partial p_{\theta}}=\frac{1}{R^{2}},
\end{gathered}
$$




$$
\frac{\partial \dot{p_{\theta}}}{\partial \theta}=-\frac{l_{z}^{2}}{R^{2} \sin ^{2} \theta}\left(1+\frac{3 \cos ^{2} \theta}{\sin ^{2} \theta}\right)+\frac{6 e^{2}}{10 R^{3}}\left(-\sin ^{2} \theta+\cos ^{2} \theta\right),
$$

então (2.50) é equivalente a

$$
\left\{\begin{array}{l}
\dot{\Theta}=\frac{P_{\Theta}}{R^{2}(t)} \\
\dot{P_{\Theta}}=-\frac{1}{R^{2}(t)}\left(l_{z}^{2}+\frac{6 e^{2}}{10 R(t)}\right) \Theta .
\end{array}\right.
$$

Tomando $s$ como variável temporal, encontramos que

$$
\begin{aligned}
& \frac{\partial \Theta}{\partial s}=\frac{\partial \Theta}{\partial t} \frac{\partial t}{\partial s}=\frac{P_{\Theta}}{R^{2}(s)} R^{2}(s)=P_{\Theta} \\
& \frac{\partial P_{\Theta}}{\partial s}=\frac{\partial \Theta}{\partial t} \frac{\partial t}{\partial s}=-\frac{1}{R^{2}(s)}\left(l_{z}^{2}+\frac{6 e^{2}}{10 R(s)}\right) \Theta R^{2}(s) \\
& =-\left(l_{z}^{2}+\frac{6 e^{2}}{10 R(s)}\right) \Theta
\end{aligned}
$$

e, portanto, (2.54) é equivalente a

$$
\partial_{s}^{2} \Theta+\left(l_{z}^{2}+\frac{6 e^{2}}{10 R(s)}\right) \Theta=0 .
$$

Substituindo a solução (2.47), finalmente encontramos que

$$
\partial_{s}^{2} \Theta+\left(\kappa-\frac{U_{0}}{\cosh ^{2}(\zeta s)}\right) \Theta=0
$$

onde

$$
\kappa=l_{z}^{2}+\frac{6 e^{2}}{10 R_{-}} \text {e } \quad U_{0}=\frac{6 e^{2}\left(\bar{R}-R_{-}\right)}{10 \bar{R} R_{-}} .
$$

Utilizando as relações (2.27) encontramos que

$$
\begin{aligned}
U_{0} & =\frac{6 e^{2}\left(\bar{R}-R_{-}\right)}{10 \bar{R} R_{-}} \\
& =\frac{6 \bar{R} R_{-}^{2}|h|\left(\bar{R}-R_{-}\right)}{\bar{R} R_{-}} \\
& =12 \zeta^{2}
\end{aligned}
$$

e, além disso,

$$
\begin{aligned}
\kappa & =l_{z}^{2}+\frac{6 e^{2}}{10 R_{-}} \\
& =l_{z}^{2}+6|h| \bar{R} R_{-} \\
& =l_{z}^{2}+\left(6|h| \bar{R} R_{-}-6|h| R_{-}^{2}\right)+6|h| R_{-}^{2} \\
& =l_{z}^{2}+12 \zeta^{2}+6|h| R_{-}^{2} .
\end{aligned}
$$


Assim, a equação (2.58) nada mais é do que a equação de Schrödinger unidimensional, homogênea no tempo e com barreira potencial $U_{0} / \cosh ^{2}(\zeta s)$ para uma partícula não relativística com energia $\kappa>U_{0}$.

Tomando uma normalização adequada, o comportamento assintótico da solução será $\Theta(s) \rightarrow C e^{i \kappa s}+B e^{-i \kappa s}$ quando $s \rightarrow-\infty$ e $\Theta(s) \rightarrow e^{i \kappa s}$ quando $s \rightarrow \infty$ Para encontrar $\alpha$, devemos calcular $|B|$ (veja [LL81]), que será dado por:

$$
|B|=\frac{\cosh \left(\frac{\pi}{2} \sqrt{\frac{4 U_{0}}{\zeta^{2}}-1}\right)}{\sinh \left(\pi \frac{\sqrt{\kappa}}{\zeta}\right)} .
$$

Mas, utilizando novamente as relações (2.27), as definições (2.59) de $U_{0}$ e $\kappa,(2.42)$ de $\zeta$ e (2.30) de $\gamma$, obtemos

$$
\begin{gathered}
\frac{4 U_{0}}{\zeta^{2}}=\frac{6 e^{2}}{5 \bar{R} R_{-}^{2}|h|}=12 \\
\frac{\sqrt{\kappa}}{\zeta}=\frac{\sqrt{l_{z}^{2}+\frac{3 e^{2}}{5 R}}}{\sqrt{\frac{1}{2} R(\bar{R}-R)|h|}}=\sqrt{\frac{2 l_{z}^{2} R+6 e^{2} / 5}{R^{2}(\bar{R}-R)|h|}}=2 \gamma
\end{gathered}
$$

e assim

$$
|B|=\frac{\cosh (\sqrt{11} \pi / 2)}{\sinh (2 \pi \gamma)}
$$

Finalmente, $\alpha$ será dado por

$$
\alpha=|B|+\sqrt{1+\left|B^{2}\right|} \geqslant 1
$$

\subsubsection{A estabilidade de $\Gamma_{h}$}

Definimos a seção de Poincaré $\Sigma$ da seguinte forma

$$
\Sigma=\left\{\left(R_{-}, p_{R}, \theta, p_{\theta}\right) ; p_{R}>0\right\} .
$$

Então, sendo $\Sigma_{E}$ a restrição de $\Sigma$ ao nível energético $E$, usando a conservação da energia, podemos tomar

$$
p_{R}=\left[2\left(E-\frac{l_{z}^{2}}{2 R_{-}^{2} \sin ^{2} \theta}+\frac{1}{R_{-}}+\frac{e^{2}\left(1-\cos ^{2} \theta\right)}{10 R_{-}^{3}}\right)-\frac{p_{\theta}^{2}}{R_{-}^{2}}\right]^{1 / 2}
$$

e utilizar

$$
y_{1}=\theta-\pi / 2 \quad \text { e } \quad y_{2}=p_{\theta}
$$

como coordenadas em $\Sigma_{E}$. Desta forma, $\mathbf{0}=(0,0) \in \Sigma_{E}$ representa o ponto $\left(R_{-},\left[2 E-l_{z}^{2} / R_{-}^{2}+2 / R_{-}+2 e^{2} / 10 R_{-}^{3}\right]^{1 / 2}, \pi / 2,0\right)$ do espaço de fases, que é, justamente, a interseção da órbita $\Gamma_{E} \subset \Delta$ com $\Sigma$.

Suponha $E=h$, então, (2.10) é equivalente a

$$
F_{h}(\mathbf{y})=A R(c+\gamma \ln 2-\gamma \ln |\omega|-2 \gamma \ln \|\mathbf{y}\|) \mathbf{y} .
$$


Seja $k=c+\gamma \ln 2-\gamma \ln |\omega|$. Neste caso, este mapa apresenta a seguinte propriedade:

$$
\begin{aligned}
F_{h}\left(e^{\pi / \gamma} \mathbf{y}\right) & =A R(k) R\left(-2 \gamma \ln \left\|e^{\pi / \gamma} \mathbf{y}\right\|\right) e^{\pi / \gamma} \mathbf{y} \\
& =A R(k) R(-2 \pi-2 \gamma \ln \|\mathbf{y}\|) e^{\pi / \gamma} \mathbf{y} \\
& =e^{\pi / \gamma} A R(k) R(-2 \gamma \ln \|\mathbf{y}\|) \mathbf{y} \\
& =e^{\pi / \gamma} F_{h}(\mathbf{y}) .
\end{aligned}
$$

Note que para $\alpha=1, F_{h}$ é apenas um twist map e todo círculo centrado em $\mathbf{0}$ é invariante, assim, 0 é estável. Embora $F_{h}$ não seja diferenciável na origem, fora dela tal mapa é analítico e pode-se utilizar o teorema KAM para mostrar que se $\alpha$ é suficientemente próximo a 1, então existem curvas fechadas ao redor de $\mathbf{0}$ invariantes por $F_{h}$. Mas, então, por (2.71), podemos encontrar infinitas outras curvas que acumulam em $\mathbf{0}$, que é, portanto, estável sob ação de $F_{h}$.

Tal resultado, junto ao teorema de instabilidade do laço encontrado em [Rag97b], levou à conjectura de que existe uma curva no espaço paramétrico de $\gamma$ e $\alpha$ tal que para pares $(\gamma, \alpha)$ de parâmetros abaixo (acima) dessa curva, existem (não existem) círculos invariantes por $F_{h}$ ao redor de $\mathbf{0}$, que é, portanto, estável (instável) sob ação de tal mapa. Resultados numéricos obtidos por Ragazzo em [Rag97a] e Figueiredo et al. [dFRM98] reforçam tal conjectura. De fato, estima-se que a curva seja aproximadamente dada por

$$
\gamma\left(\alpha-\alpha^{-1}\right)=2^{-1 / 2}
$$

ou, equivalentemente, em função de $|B|$,

$$
\gamma|B|=2^{-3 / 2}
$$

Substituindo o valor encontrado em (2.65) na equação acima, obtemos:

$$
\frac{\gamma \cosh (\sqrt{11} \pi / 2)}{\sinh (2 \pi \gamma)}=2^{-3 / 2} .
$$

Na Fig.(2.5) a linha sólida é o gráfico de $f(\gamma)=\gamma|B|$, enquanto que a curva pontilhada é o gráfico de $g(\gamma)=2^{-3 / 2}$. Assim, podemos concluir que $\mathbf{0}$ será instável para $\gamma<p$ e estável para $\gamma>p$, onde $p$ é tal que $f(p)=2^{-3 / 2}$.

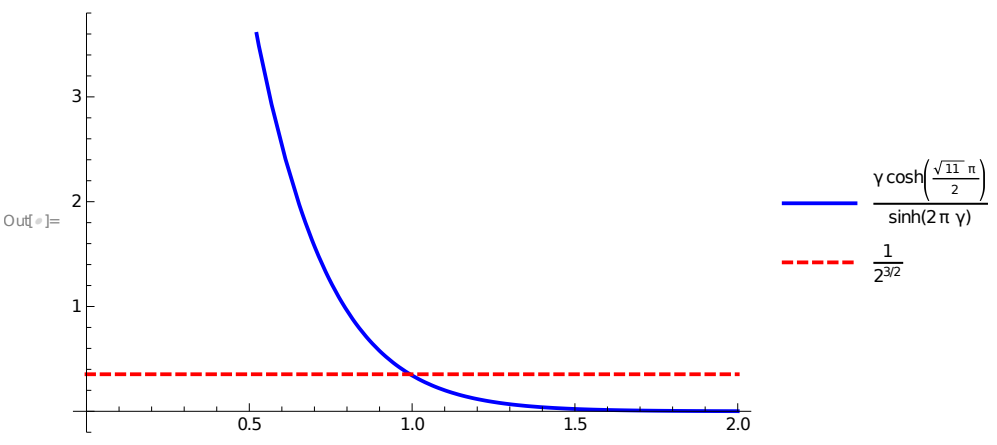

Figura 2.5: Os gráficos de $f(\gamma)=\gamma|B|$ e $g(\gamma)=2^{-3 / 2}$.

Encontramos numericamente o valor aproximado de $p=0.99362<1$. Mas note que se

$$
\gamma=\frac{1}{2} \sqrt{\frac{2 l_{z}^{2} R_{-}+6 e^{2} / 5}{R_{-}^{2}\left(\bar{R}-R_{-}\right)|h|}},
$$


então o radicando

$$
\begin{aligned}
\frac{2 l_{z}^{2} R_{-}+6 e^{2} / 5}{R_{-}^{2}\left(\bar{R}-R_{-}\right)|h|} & =\frac{2 l_{z}^{2} R_{-}-6 e^{2} / 5}{R_{-}^{2}\left(\bar{R}-R_{-}\right)|h|}+\frac{24 e^{2}}{10 R_{-}^{2}\left(\bar{R}-R_{-}\right)|h|} \\
& =-4+\frac{24 e^{2}}{10 R_{-}^{2}\left(\bar{R}-R_{-}\right)|h|} \\
& =-4+\frac{24 \bar{R}}{\left(\bar{R}-R_{-}\right)} \\
& >-4+20=16 .
\end{aligned}
$$

Assim, $\gamma>1$ e, portanto, $\mathbf{0}$ é estável sob ação de $F_{h}$ para qualquer valor de $e$.

Destacamos que a estabilidade de $\mathbf{0}$ por $F_{h}$ não implica na estabilidade do laço homoclínico como um todo. Isso se dá pela existência de pontos na variedade instável do equilíbrio centro-sela que não pertencem ao laço homoclínico. A trajetória com condição inicial $\left(R_{-},-\delta, \pi / 2,0\right)$, por exemplo, escapa de qualquer bola centrada em $\mathbf{R}_{-}$.

Sendo assim, dizemos que o laço é fracamente estável, ou seja, estável apenas sob perturbações distantes de $\mathbf{R}_{-}$.

\subsubsection{A estabilidade de 0 por $F_{E} \neq F_{h}$}

Quando $E \neq h$, a análise da estabilidade de $\mathbf{0}$ sob ação de $F_{E}$ passa a ser uma tarefa muito mais simples, mesmo quando $\alpha>1$. Neste caso, $F_{E}$ é diferenciável na origem e a estabilidade estará definida pelo traço e determinante do jacobiano. Considerando (2.13), calculamos:

$$
D F_{E}(\mathbf{0})=\left(\begin{array}{cc}
\alpha \cos v & -\alpha \sin v \\
\alpha^{-1} \sin v & \alpha^{-1} \cos v
\end{array}\right),
$$

onde $v=c-\gamma \ln |E-h|$. Portanto,

$$
\operatorname{det}\left(D F_{E}(\mathbf{0})\right)=1
$$

e

$$
\operatorname{tr}\left(D F_{E}(\mathbf{0})\right)=\left(\alpha+\alpha^{-1}\right) \cos (c-\gamma \ln |E-h|) .
$$

Uma condição suficiente e necessária para que $\mathbf{0}$ seja estável é

$$
\left|\operatorname{tr}\left(D F_{E}(\mathbf{0})\right)\right|<1-\operatorname{det}\left(D F_{E}(\mathbf{0})\right),
$$

ou seja

$$
\left|\left(\alpha+\alpha^{-1}\right) \cos (c-\gamma \ln |E-h|)\right|<2 .
$$

Isso sugere um curioso comportamento quando $E$ se aproxima de $h$. Neste caso, se $\alpha \neq 1$, o valor de $\operatorname{tr}(A R(\mathbf{0}))$ oscila continuamente no intervalo $\left[-\alpha-\alpha^{-1}, \alpha+\alpha^{-1}\right]$, fazendo com que $\mathbf{0}$ seja ora estável, ora instável. O único caso em que tal transição não ocorre é quando $\alpha=1$. Ou seja, mesmo que o laço homoclínico seja estável, em qualquer vizinhança, é possível encontrar órbitas instáveis, porém, com domínio de instabilidade muito pequeno. Tal resultado já havia sido obtido anteriormente por Churchill et al. [CPD80].

\subsubsection{Resultados numéricos}

Nesta seção utilizamos ferramentas numéricas para ilustrar os dois principais resultados obtidos ao longo do capítulo. Em 2.2.4 buscamos evidenciar a semelhança entre a função de recorrência de Poincaré $f$ - obtida integrando numericamente as equações 2.17 - e a aproximação $F$, dada por 2.10. Finalmente, em 2.2.4 exibimos a restrição da seção de poincaré a um nível de energia tal que $\left|\operatorname{tr}\left(F_{E}(0)\right)\right|>2$ e iteramos condições iniciais pelo mapa $F_{E}$. 


\section{Função de primeiro retorno e sua aproximação}

Na Fig.(2.6) vemos o resultado de iterações de $f_{E}$ (na direita) e $F_{E}$ (na esquerda) sob um conjunto de condições iniciais contidas em $\Sigma_{E}$. O nível de energia escolhido para tal simulação foi $E=h-0.01 \approx-0.4227$.
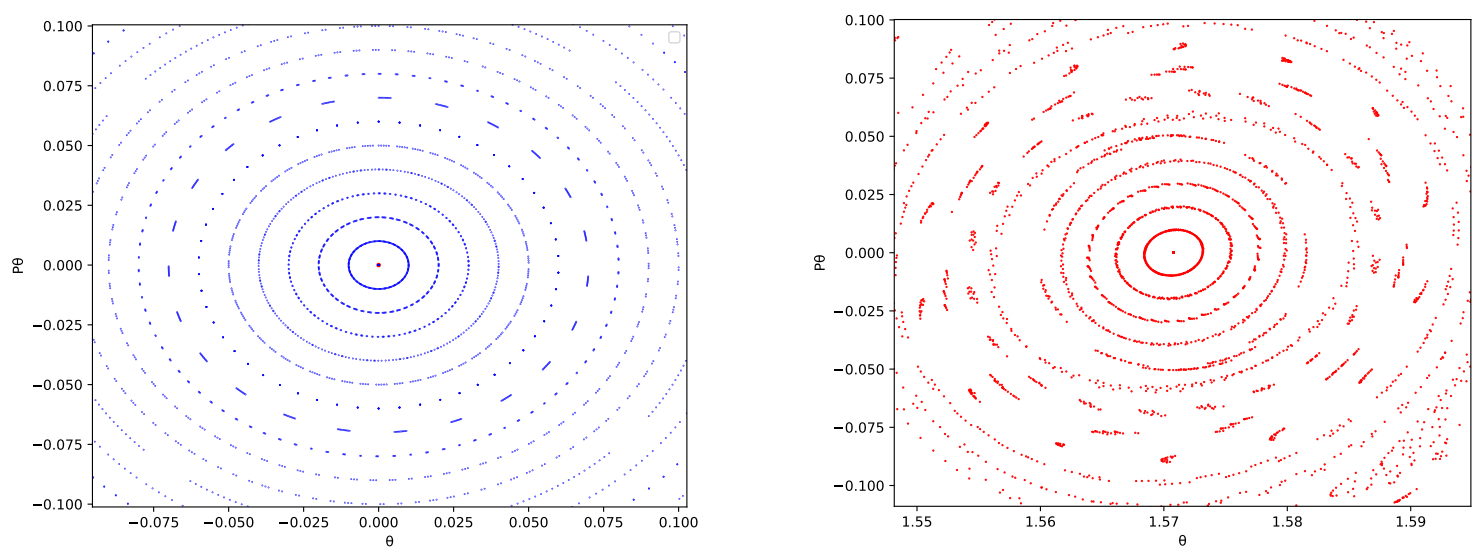

Figura 2.6: Iterações pelos mapas $F_{E}$ (esquerda) e $f_{E}$ (direita) de condições iniciais tomadas sob o eixo $\theta=\pi / 2$ da seção de Poincaré $\Sigma_{E}$. Parâmetros utilizados: $E \approx-0.4227, c=551 \pi / 360, e^{2}=0.9, l_{z}^{4}=7.9 e^{2} / 5$.

Nota-se na figura que, qualitativamente, a órbita das condições iniciais por iterações de $F_{E}$ e $f_{E}$ têm o mesmo comportamento, principalmente em regiões próximas à origem. Estimar quantitativamente essas regiões não é uma tarefa fácil. Isso se dá principalmente pela dificuldade em ajustar a constante $c$ de $F$ para o nosso problema.

Em casos de outros hamiltonianos onde a estabilidade de $\mathbf{0}$ por $F_{h}$ depende dos parâmetros do sistema, o ajuste de $c$ pode ser feito, por exemplo, comparando os resultados obtidos por iterações de $F_{h}$ e $f_{h}$ para o mesmo conjunto de condições iniciais com os parâmetros próximos à região crítica do espaço paramétrico.

Vale destacar que a diferença entre os dois retratos se dá somente pela mudança de coordenadas descrita em (2.69).

\section{Perda da estabilidade da origem de $\Sigma_{E}$}

Aqui ilustramos as iterações de um conjunto de condições iniciais sob ação do mapa $F_{E}$ para um dos casos onde $E$ é tal que $\left|\operatorname{tr}\left(D F_{E}(0)\right)\right|>2$. Como visto anteriormente, neste caso, a origem deixa de ser estável. O domínio de instabilidade, porém, é muito pequeno.

Embora suspeitemos que ele de fato esteja presente, não foi possível observar o mesmo comportamento em $f_{E}$. Estima-se numericamente por $F_{E}$, que esse fenômeno seja sensível para variações na energia da ordem de $10^{-8}$ no intervalo entre $(h-0.01, h-0.02)$. Assim, sem um valor ideal para $c$, encontrar tal comportamento será uma tarefa de busca por exaustão, que foge do escopo do trabalho. 


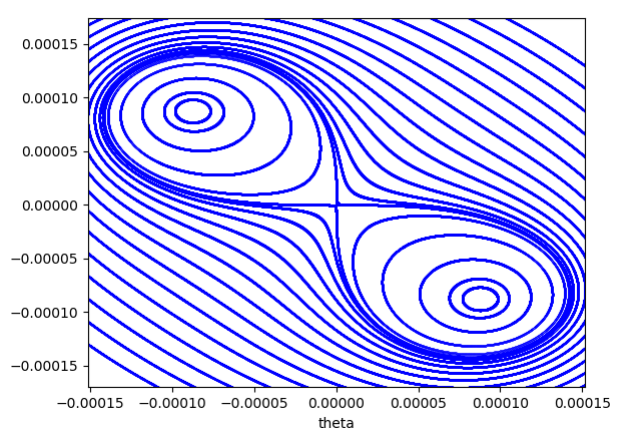

Figura 2.7: Iterações pelo mapa $F_{E}$ de condições iniciais tomadas sob o eixo $\theta=\pi / 2$ da seção de Poincaré $\Sigma_{E}$. Parâmetros utilizados: $E=h-e^{((c-5 \pi) / \gamma)}, c=551 \pi / 360, e^{2}=0.9, l_{z}^{4}=7.9 e^{2} / 5$.

\subsubsection{Escape}

Na Fig.(2.8) vemos as iterações de um conjunto de condições iniciais sob ação do mapa $f_{E}$ quando $E=h+0.01$. Como visto anteriormente, neste caso, existe uma região onde as órbitas escapam da barreira potencial. Tal região é representada, em $\Sigma_{E}$ pelo círculo branco próximo a origem. A existência de curvas invariantes garantirá que nenhuma órbita externa a curva caia na região central sob iterações de $f_{E}$.

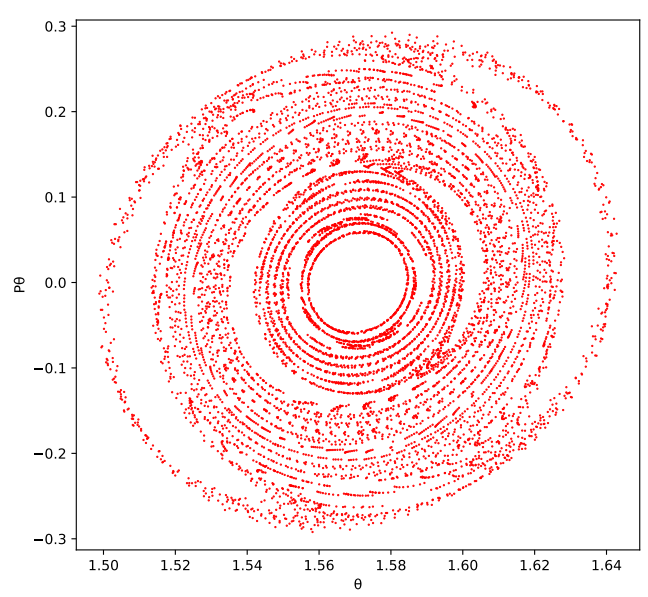

Figura 2.8: Iterações pelos mapa $f_{E}$ de condições iniciais tomadas sob o eixo $\theta=\pi / 2$ da seção de Poincaré $\Sigma_{E}$. Parâmetros utilizados: $E=-0.40273559884568557,1=10, e^{2} / 10=0.9, l_{z}^{4}=15.8 e^{2} / 101$. 


\section{Capítulo 3}

\section{Conclusões}

Iniciamos o trabalho encontrando, através da expansão de Taylor do potencial gravitacional, a fórmula geral do potencial gerado pelos termos de monopolo, dipolo e quadrupolo para uma distribuição de massa arbitrária $\rho$. A seguir, calculamos explicitamente tal potencial para uma distribuição específica, que representa um esferoide oblato homogêneo. Utilizando resultados elementares da mecânica hamiltoniana, conseguimos reduzir o problema em um grau de liberdade, reescrevendo as equações de movimento como o gradiente de um novo potencial: o potencial efetivo. Tal potencial nos fornece algumas informações qualitativas do sistema, porém, sem uma solução explícita, não é possível concluir nada a respeito de um eventual fenômeno de achatamento, a motivação central desta dissertação. Sendo assim, utilizando métodos computacionais, analisamos soluções numéricas, com as quais pudemos constatar que, em um modelo simplificado, composto apenas por uma partícula orbitando o corpo central, tal fenômeno não está presente. Porém, conjectura-se que em um cenário astronômico mais realista, onde o objeto de estudo fosse uma "núvem" de partículas, tal termo possa contribuir para o aumento da probabilidade de colisões, que por sua vez dissipam energia e fazem com que os envolvidos caiam, cada vez mais, no poço gravitacional, se acumulando do plano equatorial.

No segundo capítulo nos concentramos em estudar a dinâmica local de duas órbitas especiais do retrato de fase. A primeira delas, associada ao ponto de mínimo local do potencial efetivo restrito ao plano equatorial, é uma órbita circular do problema original. Com uma aproximação para o potencial em sua vizinhança pudemos encontrar uma solução explícita aproximada, conforme o método tradicional de pequenas oscilações. Finalizamos a seção com um comparativo entre a solução analítica da aproximação e a respectiva solução numérica. Vimos que tal aproximação é muito mais sensível para variações na coordenada $z$ e que bons resultados são encontrados com condições iniciais até a ordem de $10^{-2}$, ou equivalentemente $1 \%$ do raio equatorial do esferoide.

Na seção seguinte, investigamos a órbita associada ao ponto de máximo local do potencial efetivo quando restrito ao plano equatorial. Pudemos observar que, para determinadas condições iniciais, tal órbita é homoclínica ao ponto de equilíbrio, que por sua vez, é do tipo centro-sela. Definindo uma seção de Poincaré no espaço de fases e utilizando uma aproximação para a função de primeiro retorno, utilizamos resultados obtidos por Ragazzo em [Rag97b] para determinar a estabilidade do laço homoclínico (conjunto composto pela órbita e o equilíbrio citados acima). Encontramos que o laço é fracamente estável, ou seja, estável para perturbações distantes do equilíbrio centrosela. Apesar disso, pudemos ver que em qualquer vizinhança de tal conjunto é possível encontrar outras órbitas que são localmente instáveis que possuem domínio de instabilidade muito pequeno. Novamente, encerramos a seção com resultados numéricos que reforçam as afirmações feitas ao longo do capítulo. 
CONCLUSÕES 


\section{Referências Bibliográficas}

[CPD80] R.C Churchill, G. Pecelli e D.L.Rod. Stability Transittions for Periodic Orbits in Hamiltonian Systems. Archive for Rational Mechanics and Analysis, (73):313-347, 1980. 35

[dFRM98] J.C. Bastos de Figueiredo, C. Grotta Ragazzo e C.P. Malta. Two important numbers in the Hénon-Heiles dynamics. Phisics Lettetrs A, (241):35-40, 1998. 2, 34

[DS15] Gabriela Depetri e Alberto Saa. Chaos in the kepler problem with quadrupole perturbations. Fields Institute Communications, página 93-98, 2015. 1, 26

[LBP74] D. Lynden-Bell e J. Pringle. The evolution of viscous discs and the origin of the nebular variables. Monthly Notices of the Royal Astronomical Society, 168:603-637, 09 1974. 1

[Ler91] Lev Mikhailovich Lerman. Hamiltonian systems with loops of a separatrix of a saddlecenter. Selecta Mathematica Sovietica, 10, 01 1991. 26

[LL81] L. D. Landau e L. M. Lifshitz. Quantum Mechanics Non-Relativistic Theory, Third Edition: Volume 3. Butterworth-Heinemann, 3 edição, Janeiro 1981. 33

[LRCLS11] P. Letelier, J. Ramos-Caro e F. López-Suspes. Chaotic motion in axially symmetric potentials with oblate quadrupole deformation. Physics Letters A - PHYS LETT A, 375:3655-3658, 10 2011. 26

[MHO92] A. Mielke, P. Holmes e O. O'Reilly. Cascades of homoclinic orbits to, and chaos near, a Hamiltonian saddle-center. Journal of Dynamics and Differential Equations, (4):95-126, 1992. 26

[Mil66] D. Milder. Dynamics of flattening in rotating stellar systems. The Astrophysical Journal, 145:109, 06 1966. 1

[Pri03] James E. Pringle. Accretion discs in astrophysics. Annual Review of Astronomy and Astrophysics, 19:137-160, 11 2003. 1

[Rag97a] Clodoaldo Grotta Ragazzo. On the Stability of Double Homoclinic Loops. Communications in Mathematical Physics, (184):251-272, 1997. 34

[Rag97b] Clodoaldo Grotta Ragazzo. Stability of homoclinic orbits and diffusion in phase space. Phisics Lettetrs A, (230):183-190, 1997. 2, 26, 34, 39

[ZR01] Salvador Addas Zanata e Clodoaldo Grotta Ragazzo. Critical number in scattering and escaping problems in classical mechanics. Phisical Review E, 64, 2001. 2 